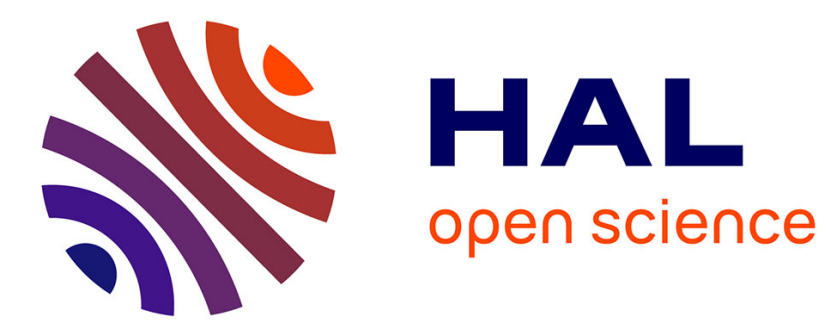

\title{
A growth-fragmentation approach for modeling microtubule dynamic instability
}

Stephane Honore, Florence Hubert, Magali Tournus, Diana White

\section{To cite this version:}

Stephane Honore, Florence Hubert, Magali Tournus, Diana White. A growth-fragmentation approach for modeling microtubule dynamic instability. Bulletin of Mathematical Biology, 2019, 81 (3), pp.722758. 10.1007/s11538-018-0531-2 . hal-01630658

\section{HAL Id: hal-01630658 https://hal.science/hal-01630658}

Submitted on 7 Nov 2017

HAL is a multi-disciplinary open access archive for the deposit and dissemination of scientific research documents, whether they are published or not. The documents may come from teaching and research institutions in France or abroad, or from public or private research centers.
L'archive ouverte pluridisciplinaire HAL, est destinée au dépôt et à la diffusion de documents scientifiques de niveau recherche, publiés ou non, émanant des établissements d'enseignement et de recherche français ou étrangers, des laboratoires publics ou privés. 


\title{
A growth-fragmentation approach for modeling microtubule dynamic instability
}

\author{
Florence Hubert * $\quad$ Magali Tournus ${ }^{\dagger} \quad$ Diana White ${ }^{\ddagger}$
}

\begin{abstract}
Microtubules (MTs) are protein filaments found in all eukaryotic cells which are crucial for many cellular processes including cell movement, cell differentiation, and cell division. Due to their role in cell division, they are often used as targets for chemotherapy drugs used in cancer treatment. Experimental studies of MT dynamics have played an important role in the development and administration of many novel cancer drugs, however, a complete description of MT dynamics is lacking. Here, we propose a new mathematical model for MT dynamics, that can be used to study the effects of chemotherapy drugs on MT dynamics. Our model consists of a growth-fragmentation equation describing the dynamics of a density of MTs, coupled with two ODEs that describe the dynamics of free GTP- and GDP-tubulin concentrations (the individual dimers that comprise of MTs). We prove the well-posedness of our system and perform a numerical exploration of the influence of the parameters on the systems dynamics. This exploration helps provide insight into the action of MT-targeting chemotherapy drugs on MT dynamics.
\end{abstract}

Key-words: Growth-fragmentation model, Banach fixed point, Microtubules dynamics. Subject Classifications: 45K05, 92C37.

\section{Introduction}

Microtubules (MTs) are dynamic protein polymers that are found in all eukaryotic cells. They are crucial for normal cell development, aiding in many cellular processes, including cell division, cell polarization, and cell motility [21]. Due to their role in cell movement and cell division, these polymers are often used as targets for a variety of cancer chemotherapy drugs. Many experimental studies have been completed to understand MT dynamics [9, 2, 23, 1], and how these dynamics are altered by the addition of MT targeting drugs [15, 26]. However, a complete understanding of such dynamics is lacking, and so the development of new theoretical models to describe MT dynamics is important.

MTs undergo a unique type of dynamics referred to as dynamic instability, which was first described by Kirschner and Mitchison in 1984 [9]. This type of dynamics is unique to MT polymers, and refers to the relative slow growth of a MT, followed by very fast depolymerization. The switch from growing to shortening is referred to as a catastrophe event, whereas the switch from shortening to growth is refered to as a rescue.

MTs are composed of tubulin heterodimers, and grow through the addition of GTP-bound tubulin (guanosine triphosphate), generally from the so called plus end of the MT, and shrink

*Aix Marseille Univ, CNRS, Centrale Marseille, I2M, Marseille, France. Email: magali.tournus@centralemarseille.fr.

${ }^{\dagger}$ Aix Marseille Univ, CNRS, Centrale Marseille, I2M, Marseille, France. Email: florence.hubert@univ-amu.fr

${ }^{\ddagger}$ Clarkson University, Department of Mathematics, Potsdam, New York. Email: dtwhite@clarkson.edu 


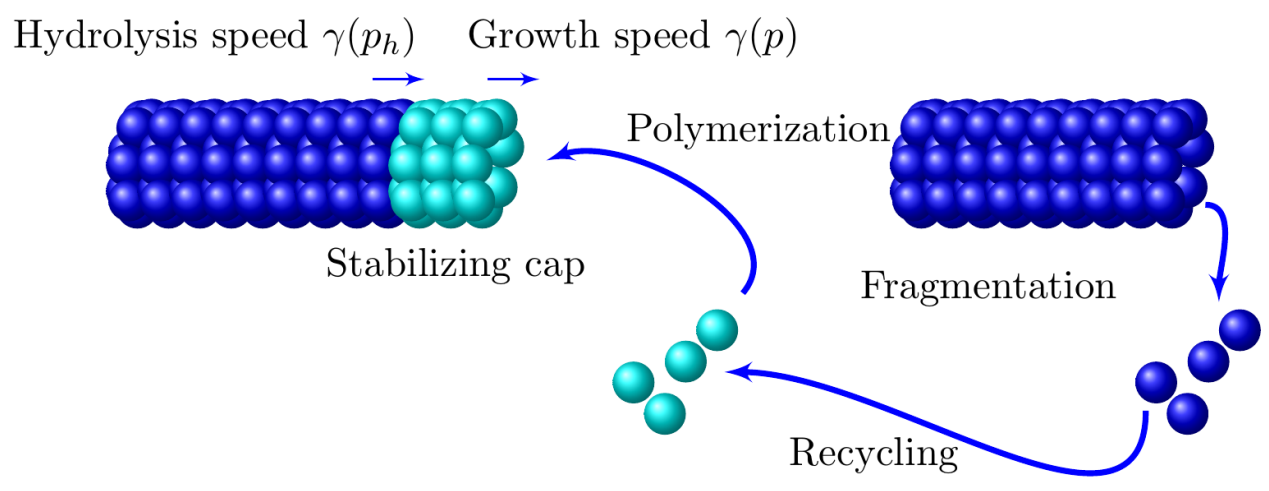

Figure 1: Schematic representation of the polymerization-fragmentation-recycling cycle. When the hydrolysis rate exceeds the growth rate, the stabilizing cap (in light blue) gradually disappears. As soon as the cap completely vanishes, a catastrophe (shortening event) can occur: the microtubule shortens and the remaining GDP-tubulin (in blue) is released into the system. GDP-tubulin is then recycled into GTP-tubulin (in light blue) which is used for the growth of other microtubules through polymerization.

through dissociation of GDP-bound tubulin (guanosine diphosphate) at this same end [21]. The minus end of the MT is generally more stable, being capped by stabilizing proteins.

As MTs grow, older GTP-tubulin dimers hydrolyze to the lower energy GDP-tubulin, creating two distinct regions along the length of a MT. That is, a back end composed of GDP-tubulin, and a front end composed of GTP-tubulin. This GTP region at the growing end of a MT is referred to as the CAP. If a MT is growing at a rate faster than that of hydrolysis, the MT will continue to grow. However, if the rate of hydrolysis is greater than the rate of growth, the CAP region will begin to shorten. If the CAP region vanishes, the MT will undergo a so-called catastrophe, and enter a state of shortening (fragmentation). See Figure 1 for a representation of the polymerizationfragmentation-recycling cycle.

Since the discovery of dynamic instability, many theoretical [3, 7], stochastic, and computational models [6, 12, 14, 13, have been developed to better understand this unique type of behavior. Most continuous models use advection-type terms to describe MT growth and shortening at the macroscopic level, whereas stochastic-type models, and many computational models provide a microscopic description of dimer addition and subtraction (see for example the first stochastic model by Hill and Chen [6]).

Here, based on the Hinow model [7, we develop a novel deterministic modeling approach in a continuous setting to describe MT dynamics. Similar to the Hinow model, we follow the mean behavior of a family of microtubules. The uniqueness of our approach is the use of a fragmentation term in the MT dynamics equation to account for sudden MT shortening. The growth-fragmentation equation is coupled to a system of ODEs that describe the dynamics of free-GTP and GDP-tubulin populations. Models consisting of an ODE coupled with an integro-partial differential equation are already extensively used and studied in the description of Prion dynamics [5]. To the best of our knowledge, the use of a fragmentation term to represent the sudden depolymerization of a microtubule has only been used by the authors [24]. One specificity of our model is the fragmentation kernel which is not self-similar, so that our system cannot be easily reduced to a system of ODEs, like was done for Prion.

In addition to modeling MT dynamic instability in the base case (the case without introduction of drugs), we account for the action of MT targeting chemotherapy drugs on MT dynamics through 
variation of certain model parameters. Such drugs work by acting on MTs during cell division, causing cells to die, and alter the dynamics of MTs by either promoting MT assembly (MT stabilizing drugs) or promoting MT dissasembly (MT destabilizing drugs). However, at low (non-cytotoxic) doses, such drugs can alter MT dynamics without significantly altering the total MT polymer mass [26]. The exploration of numerical solutions and their comparison with experimental results provides insight into which parameters are likely to be regulated by the addition of MT targeting drugs. In particular, these simulations will allow us to determine which parameter variations promote MT stabilization and which promote destabilization.

This model describes the dynamics of MTs in an in vitro setting. Describing the dynamics of MTs in an in vivo setting would require us to take into account many other cellular components, such as MT associated proteins and other cellular components including the cell boundary [21].

The outline of the paper is the following: In Section 2, we detail the development of our model which consists of an integro-partial differential equation, endowed with biologically realistic boundary conditions to describe MT dynamics, coupled with two ODEs that describe the time evolution of free GTP and GDP-tubulin concentrations. Here, we provide specific properties that certain

model functions and parameters must satisfy. Section 3 is devoted to the well-posedness of the model. Here, we place more general assumptions on model parameters and functions. Finally, in Sections 4, we perform numerical simulations to illustrate the behavior of our model in the base case. Parameters are estimated so that certain outputs of the model fit experimental data. In this section we also incorporate the action of MT targeting drugs through alteration of certain model parameters. Using these results we explore how stabilizing and destabilizing drugs might work to alter the normal (base case) behavior of the MT/tubulin system.

\section{Description of the model}

The evolution of the density $u(x, t)$ of microtubules of length $x \geq 0$ at time $t>0$ is described by the one-dimensional growth-fragmentation equation (1). We neglect the complex cylindrical shape of MTs, and assume that they have linear structures.

$$
\frac{\partial u(x, t)}{\partial t}+\gamma(p(t)) \frac{\partial u(x, t)}{\partial x}=\beta(p(t))\left(-\int_{0}^{x} k(x, y) d y u(x, t)+\int_{x}^{\infty} k(y, x) u(y, t) d y\right)+N(p(t)) \xi(x)
$$

Equation (1) is coupled with a system of two ODEs, describing the time evolution of the concentrations of GTP-tubulin $p(t)$ and GDP-tubulin $q(t)$, respectively,

$$
\begin{gathered}
\frac{d p}{d t}(t)=-\gamma(p(t)) \int_{0}^{\infty} u(x, t) d x-N(p(t))+\kappa q(t), \\
\frac{d q}{d t}(t)=\beta(p(t)) \int_{0}^{\infty} u(y, t) \int_{0}^{y}(y-x) k(y, x) d x d y-\kappa q(t),
\end{gathered}
$$

endowed with initial conditions $u(x, 0)=0, q(0)=0$, and $p(0)=p_{0}>0$, and with the boundary condition $u(0, t)=0$.

The transport term of equation (1) describes the growth of MTs at rate $\gamma(p)$ which depends on the free GTP-tubulin concentration, since experiments suggest that MTs undergo periods of growth that are dependent on this concentration. An example of $\gamma(p)$, which is similar to growth curves found in experiment [11], is given by formula (4) 


$$
\gamma(p)= \begin{cases}0 & \text { for } p<p_{c} \\ \alpha\left(p-p_{c}\right) & \text { for } p_{c}<p<p_{\infty} \\ \alpha\left(p_{\infty}-p_{c}\right) & \text { for } p_{\infty}<p\end{cases}
$$

where $p_{c}$ represents the critical concentration required to initiate MT growth, $p_{\infty}$ the saturation polymer concentration, and the growth rate parameter $\alpha>0$.

The main novelty of the model is the assumption that depolymerization occurs instantaneously, and not at a finite rate. The integral term of equation (1) describes a shortening event. We consider that such an event has a chance to occur when the fixed rate of hydrolysis $\gamma^{h}$ exceeds the rate of growth $\gamma(p(t))$ of a MT. We assume $p_{\infty}$ large enough so that $\alpha\left(p_{\infty}-p_{c}\right)>\gamma^{h}$, and then, $\gamma(p)<\gamma^{h} \Leftrightarrow p<\left(\gamma^{h}+\alpha p_{c}\right) / \alpha:=p_{h}$. If $p$ falls below the value $p_{h}$, MTs will have a chance to shorten. However, if $p$ stays above the value $p_{h}$, there is no MT shortening. We introduce the parameter $\beta(p)$ defined as

$$
\beta(p)=\beta_{\infty}\left(1-H\left(p, p_{h}\right)\right), \quad H\left(p, p_{h}\right)=\frac{1}{2}\left(1+\tanh \left(c\left(p-p_{h}\right)\right)\right),
$$

where $\beta_{\infty}$ denotes the maximal shortening rate of MTs and $H\left(p, p_{h}\right)$ is a smooth approximation to a heavy-side function. Here, $c$ describes the steepness of the transition between 0 and $\beta_{\infty}$ (i.e., the larger the value of $c$, the steeper the transition). The fact that the function $\beta$ is smooth is a technical assumption that we need in Section 3.

The function $k(y, x)$ from equation (1) represents the rescaled probability that a MT of size $y$ shortens to a MT of size $x<y$, where the remainder of the MT completely depolymerizes into GDP-tubulin dimers. Biological observations (see Figure 2) of such shortening events point out two different cases: either MTs of length $y$ shorten by an average fixed length $x_{0}$ [16 and then give rise to a newborn MT of average length $y-x_{0}$, or MTs of length $y$ shorten to give rise to newborn MTs of average fixed size $x_{0}$. These two cases help define the following two shortening kernels, $k_{0}$ and $k_{1}$, respectively:

$$
k_{0}(y, x)=G(y-x), \quad k_{1}(y, x)=G(x), \quad G(z)=\frac{1}{\sigma \sqrt{2 \pi}} \exp \frac{-\left(z-x_{0}\right)^{2}}{2 \sigma^{2}}, \quad x_{0}>0, \sigma>0 .
$$

In Figure 2 (left), we show a single kymograph, illustrating the growth dynamics of a single MT over time (taken from the experiment of Pagano et al.[16]). A kymograph describes the growth trajectory of a single MT, and is read from top to bottom. In the experiment [16], all MTs shorten by approximately the same distance $x_{0}$. In particular, the shortening distances can be fit to a Gaussian like $k_{0}$, where the standard deviation $\sigma$ is very small. Possible values for $x_{0}$ and its standard deviation $\sigma$ are summarized in Table 1 ,

The final source term in equation (1), $N(p)$, describes MT nucleation (the birth of a MT). We assume

$$
N(p)=\mu p^{m} H\left(p, p_{N}\right),
$$

where $H\left(p, p_{N}\right)$ is defined as in equation (5). In particular, equation (7) states that if the value for GTP-tubulin $(p)$ falls below the critical nucleation value $p_{N}$, nucleation is switched off. Here, $\mu$ is called the nucleation parameter and $m$ is linked to the minimum number of GTP-tubulin dimers required for nucleation. Freshly nucleated MTs have a size between 0 and $x_{\text {min }}$. The weight $\xi(x)$ is then defined as 

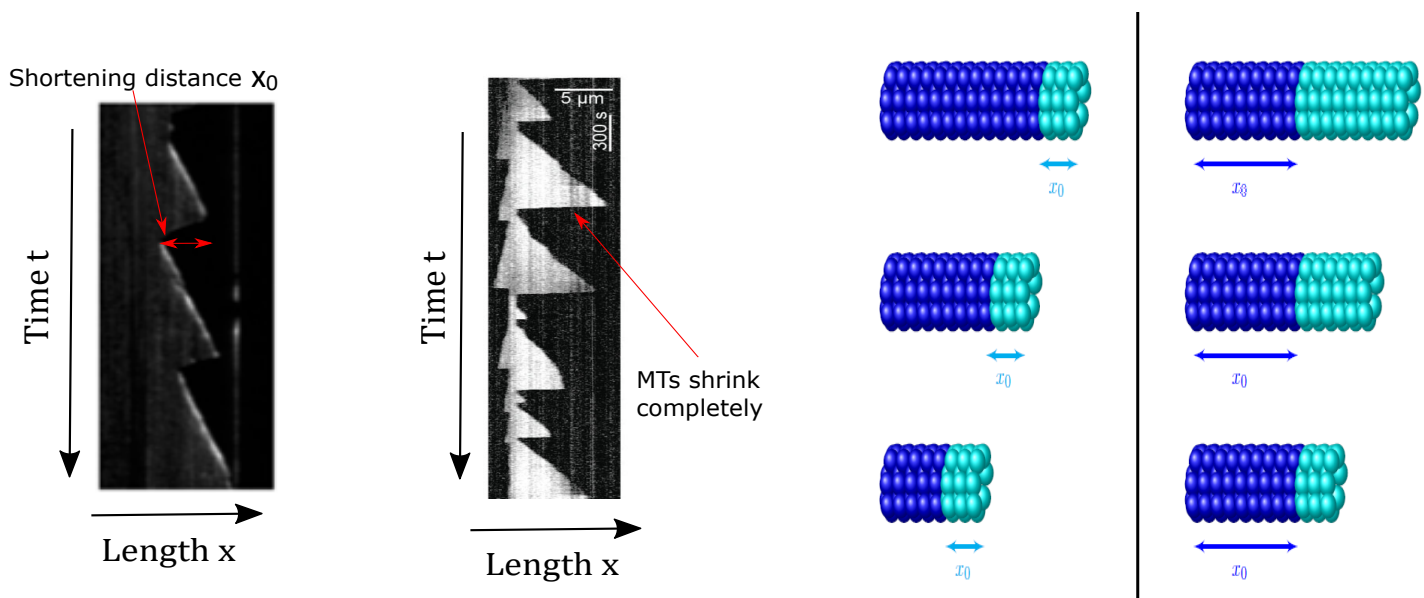

Figure 2: Examples of the growth-fragmentation process. Left: A kymograph showing a MT that shortens by approximately the same size $x_{0}$ (the appropriate kernel is $k_{0}$ ) Center: A kymograph showing a MT that shortens to the same size $x_{0}$ (the appropriate kernel is $k_{1}$ ). The experimental conditions underlying these Kymographs are described in [16]. Right: Schematic representation of the two types of kernels, $k_{1}$ and $k_{0}$, respectively.

$$
\xi(x)=C_{N}\left(1-H\left(x, x_{\min }\right)\right) .
$$

Here $x_{\min }$ is defined in Table 1 and $C_{N}$ is a normalization constant that ensures we have the property

$$
\int_{0}^{\infty} \xi(x) x d x=1
$$

Equations (2) and (3) represent the time evolution of GTP and GDP-tubulin, respectively. The first term in equation (2) describes removal of GTP tubulin due to MT growth, while the second term describes removal due to nucleation. The final term describes GDP/GTP recycling, where $\kappa>0$ is the recycling rate of GDP to GTP tubulin. The first term in equation (3) describes all GDP-tubulin which comes from a shortening event, while the second term accounts for GDP/GTP recycling.

It can be shown by formal integration that the total mass of the system (1), (2), (3) is preserved. Specifically, the total amount of tubulin in polymer and free form does not vary with time so that

$$
\frac{d}{d t}\left(\int_{0}^{\infty} x u(x, t) d x+p(t)+q(t)\right)=0 .
$$

It can also be shown that increase in the total number of MTs is only due to nucleation. That is,

$$
\frac{d}{d t}\left(\int_{0}^{\infty} u(x, t) d x\right)=N(p(t)) \int_{0}^{\infty} \xi(x) d x
$$

\section{Well posedness of the PDE model}

In this section of the paper, we focus on the well-posedness of the model developed in Section 2 . To avoid cumbersome calculations, we reduce the system defined by equations (1), (2), and (3) to a system of two equations. In particular, we assume that the exchange rate of GDP-tubulin to GTP-tubulin is instantaneous, and so we need only to consider the equations for $u$ and $p$. The 
generalization of the results that follow, to the full system, is straightforward. The PDE model we consider is written as

$$
\begin{gathered}
\frac{\partial u(x, t)}{\partial t}+\gamma(p(t)) \frac{\partial u(x, t)}{\partial x}=\beta(p(t))\left(-\int_{0}^{x} k(x, y) d y u(x, t)+\int_{x}^{\infty} k(y, x) u(y, t) d y\right)+N(p(t)) \xi(x), \\
\frac{d p}{d t}(t)=-\gamma(p(t)) \int_{0}^{\infty} u(x, t) d x+\beta(p(t)) \int_{0}^{\infty} u(y, t) \int_{0}^{y}(y-x) k(y, x) d x d y-N(p(t)), \\
u(x, 0)=0, \quad u(0, t)=0, \quad p(0)=p_{0} .
\end{gathered}
$$

A similar system was introduced and developed in the context of Prion proliferation. Global existence and uniqueness of a global solution was then studied in [10] [22] [20] using semi-group theory. The main difference between our model and that studied in [10] and [22] are the time-dependence of the function $\beta$ and the fact that the fragmentation kernel is not self-similar.

\subsection{Assumptions on the parameters}

Here, we outline assumptions on model parameters required for our main result. The polymerization growth rate $\gamma(p)$ is Lipchitz and bounded so that

$$
0 \leq \gamma\left(p_{1}\right) \leq \gamma_{\infty}, \quad\left|\gamma\left(p_{1}\right)-\gamma\left(p_{2}\right)\right| \leq \alpha\left|p_{1}-p_{2}\right|, \quad p_{1}, p_{2} \in \mathbb{R}^{+} .
$$

The nucleation density satisfies

$$
\begin{gathered}
\xi \in C^{1}\left(\mathbb{R}^{+}\right), \quad \int_{0}^{\infty} \xi(x) x d x=1, \quad \operatorname{Supp}(\xi) \subset\left[x_{m}^{\xi}, x_{M}^{\xi}\right], \quad I_{\xi}:=\int_{0}^{\infty} \xi(x) d x<\infty \\
0 \leq N(p) \leq N_{\infty}, \quad p \in \mathbb{R}^{+} .
\end{gathered}
$$

The rate of fragmentation satisfies

$$
\beta \in L^{\infty}\left(\mathbb{R}^{+}\right) \cap C^{1}\left(\mathbb{R}^{+}\right), \quad 0 \leq \beta(p) \leq \beta_{\infty}, \quad p \in \mathbb{R}^{+} .
$$

All parameters satisfy $\gamma(p)=N(p)=\beta(p)=0$ for $p<0$. The fragmentation rate is bounded such that

$$
\int_{0}^{y} k(y, x) d x=B(y), \quad B \in L^{\infty}\left(\mathbb{R}^{+}\right)
$$

where the kernel $k$ satisfies

$$
k(x, x)=0, x \in \mathbb{R}^{+}, \quad k(x, 0)=0, x \in \mathbb{R}^{+}, \quad k(x, y)=0 \text { if } x<y, \quad x, y \in \mathbb{R}^{+},
$$

and we assume the additional properties

$$
\int_{0}^{y} k(y, x)(y-x) d x \leq M_{0}+M_{1} y, \quad \int_{0}^{y}\left|\partial_{1} k(y, x)\right| d x \leq M_{2}, \quad \int_{0}^{y}\left|\partial_{2} k(y, x)\right| d x \leq M_{3} .
$$

Two different kernels are considered for application:

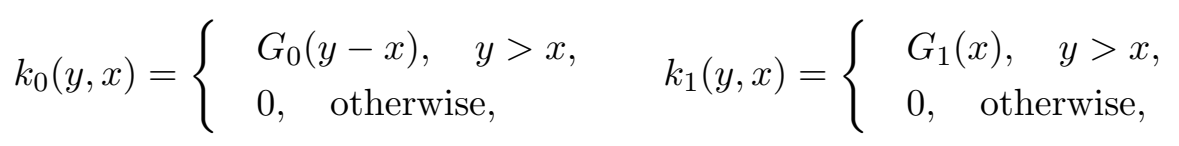

for some $G_{0}, G_{1} \in \mathcal{C}_{C}^{1}(0,+\infty)$. These two kernels correspond to the experimentally observed cases illustrated in Figure 2. The kernel $k_{0}$ satisfies properties 19 with $M_{1}=0$. In the case $M_{1}=0$, the proof of existence is easier (than when $M_{1} \neq 0$ ), thus we treat this case independently. For any $f \in L^{\infty}\left(\mathbb{R}^{+}\right)$we use the notation $f_{\infty}:=\|f\|_{L^{\infty}\left(\mathbb{R}^{+}\right)}$. 


\subsection{Main result}

Theorem 1. Under assumptions (13), (14), (15), (16), (17) (18) and (19), there exists a unique solution $(u, p) \in C\left(\mathbb{R}^{+} ; L^{1}\left(\mathbb{R}^{+},(1+x) d x\right)\right) \times C\left(\mathbb{R}^{+}\right)$to the system (10) (11). Moreover it satisfies for all $T \geq 0$

$$
\begin{gathered}
0 \leq p(T) \leq p_{0}, \quad \int_{\mathbb{R}^{+}} u(x, T) x d x \leq p_{0}, \quad \text { and } \quad \int_{\mathbb{R}^{+}} u(x, T) d x \leq N\left(p_{0}\right) T, \\
\text { and } \int_{\mathbb{R}^{+}}\left|\frac{\partial u}{\partial x}(x, T)\right| d x \leq K_{1} T+K_{2} T^{2},
\end{gathered}
$$

for some positive constants $K_{1}$ and $K_{2}$ given by

$$
K_{1}=\left(x_{M}^{\xi}-x_{m}^{\xi}\right) N\left(p_{0}\right) \xi_{\infty}^{\prime}, \quad K_{2}=\frac{\beta\left(p_{0}\right)}{2} N\left(p_{0}\right) I_{\xi}\left(M_{2}+M_{3}\right) .
$$

Strategy of the proof We build a sequence $T_{0}, T_{1}, T_{2} \ldots$ and prove by induction that the system (10) (11) admits a unique solution over $\left[0, T_{n}\right]$, and that $T_{n} \rightarrow \infty$. To do so, we assume that there exists a unique solution $\left(u^{*}, p^{*}\right)$ to the system (10), (11) over $\left[0, T_{n}\right]$. To extend this solution to $\left[T_{n}, T_{n+1}\right]$, we use the Banach fixed point theorem in the Banach space $Y=C\left(\left[T_{n}, T\right]\right)$ endowed with $\|p\|_{Y}=\sup _{T_{n} \leq t \leq T}\|p(t)\|$. For a given function $\bar{p} \in Y$, we define $u$ as the unique solution for $t \geq T_{n}$ and $x \geq 0$ to the equation

$$
\begin{gathered}
\frac{\partial u(x, t)}{\partial t}+\gamma(\bar{p}(t)) \frac{\partial u(x, t)}{\partial x}=\beta(\bar{p}(t))\left(-u(x, t) \int_{0}^{x} k(x, y) d y+\int_{x}^{\infty} k(y, x) u(y, t) d y\right)+N(\bar{p}(t)) \xi(x), \\
u\left(x, T_{n}\right)=u^{*}\left(x, T_{n}\right), \quad u(0, t)=0 .
\end{gathered}
$$

For the function $u$ given by equations (21) and (22), we then define $p:=\mathbf{G}[\bar{p}]$ as the unique solution satisfying the equation

$$
\begin{gathered}
\frac{d p}{d t}(t)=-\gamma(\bar{p}(t)) \int_{0}^{\infty} u(x, t) d x+\beta(\bar{p}(t)) \int_{0}^{\infty} u(y, t) \int_{0}^{y}(y-x) k(y, x) d x d y-N(\bar{p}(t)) \\
p\left(T_{n}\right)=p^{*}\left(T_{n}\right),
\end{gathered}
$$

for $t \geq T_{n}$ and $x \geq 0$.

We obtain the unique extent $(u, p)$ to the solution $\left(u^{*}, p^{*}\right)$ of system (10) and (11), over $\left[T_{n}, T_{n+1}\right]$ as the unique fixed point to map $\mathbf{G}$ for $p$, and the unique $u$ satisfying (21) and (22) where we replaced $\bar{p}$ by $p$. To make further notation less complicated, we drop the dependence in $n$ for the space $Y$ and the map G. The sequence of lemmas that follow provide us with the necessary details needed to extend the solution to $\left[T_{n}, T_{n+1}\right]$, and provides us with an explicit expression for $T_{n+1}$. These steps will lead us to the desired end result, the proof of Theorem 1. As a first step, we show that $\mathbf{G}$ is well defined.

\subsection{The transport-fragmentation equation}

For $a, b, c \in L^{\infty}\left(\mathbb{R}^{+}\right)$and $T_{n}>0$ given, we consider the transport-fragmentation equation for $x \geq 0$ and $t \geq T_{n}$

$$
\left\{\begin{aligned}
\frac{\partial u(x, t)}{\partial t}+a(t) \frac{\partial u(x, t)}{\partial x} & =b(t)\left(-u(x, t) \int_{0}^{x} k(x, y) d y+\int_{x}^{\infty} k(y, x) u(y, t) d y\right)+c(t) \xi(x), \\
u\left(x, T_{n}\right) & =u^{*}\left(x, T_{n}\right), \quad x \geq 0, \quad u(0, t)=0, \quad t \geq T_{n} .
\end{aligned}\right.
$$


Lemma 1 (Well posedness of (21)). Assume $a, b, c \in L^{\infty}\left(\mathbb{R}^{+}\right)$with $a \geq 0, b \geq 0, c \geq 0$, then, there exists a unique solution to 25) in $\mathcal{C}\left(\left[T_{n}, \infty\right), L^{1}\left(\mathbb{R}^{+}\right)\right)$. Moreover, we have the following properties

1. Positivity. If $u^{*}\left(., T_{n}\right) \geq 0$, then $u(., t) \geq 0$ for $t \geq T_{n}$.

2. Compact support. If for some $M>x_{M}^{\xi}, \operatorname{supp}\left(u^{*}\left(., T_{n}\right)\right) \subset\left[0, M+a_{\infty} T_{n}\right]$ (this is true for $\left.T_{n}=0\right)$, then $\operatorname{supp}(u(., t)) \subset\left[0, M+a_{\infty} t\right]$ for $t \geq T_{n}$.

Proof. The proof of Lemma 1 relies on a (second) fixed point argument. For any $\bar{u} \in C\left(\mathbb{R}^{+}, L^{1}\left(\mathbb{R}^{+}\right)\right)$ such that $\bar{u} \geq 0$, a.e., we define $u:=\mathbf{F}[\bar{u}]$ as the unique solution to

$$
\left\{\begin{aligned}
\frac{\partial u(x, t)}{\partial t}+a(t) \frac{\partial u(x, t)}{\partial x} & =b(t)\left(-B(x) u(x, t)+\int_{x}^{\infty} k(y, x) \bar{u}(y, t) d y\right)+c(t) \xi(x), \\
u\left(x, T_{n}\right) & =u^{*}\left(x, T_{n}\right), \quad x \geq 0, \quad u(0, t)=0, \quad t \geq T_{n} .
\end{aligned}\right.
$$

\section{Step 1. The map $\mathbf{F}$ is well defined.}

Based on the method of characteristics, we explain why the map $\mathbf{F}$ is well defined. The characteristic curves are defined for all time $t \geq T_{n}$ as

$$
\dot{Z}\left(t ; t_{0}, y_{0}\right)=a(t), \quad Z\left(t_{0} ; t_{0}, y_{0}\right)=y_{0},
$$

and for all $(x, t) \in \mathbb{R}^{+} \times\left[T_{n},+\infty\right)$, there exists a unique $\left(x_{0}, t_{0}\right)$ such that either $t_{0}=T_{n}$, either $x=0$ and such that $Z\left(t ; t_{0}, x_{0}\right)=x$. Let us first assume $\bar{u} \in \mathcal{C}^{1}\left(\mathbb{R}^{+} \times \mathbb{R}^{+}\right)$and $u^{*}\left(., T_{n}\right) \in \mathcal{C}^{1}\left(\mathbb{R}^{+}\right)$. The function $u$ is a solution to (26) if and only if it satisfies the linear equation

$$
\begin{aligned}
\frac{d}{d t} u\left(Z\left(t ; t_{0}, y_{0}\right), t\right)= & b(t)\left(-B\left(Z\left(t ; t_{0}, y_{0}\right)\right) u\left(Z\left(t ; t_{0}, y_{0}\right), t\right)+\int_{Z\left(t ; t_{0}, y_{0}\right)}^{\infty} k\left(y, Z\left(t ; t_{0}, y_{0}\right)\right) \bar{u}(y, t) d y\right) \\
& +c(t) \xi\left(Z\left(t ; t_{0}, y_{0}\right)\right) .
\end{aligned}
$$

There exists a unique global solution $\left.u\left(Z\left(t ; t_{0}, y_{0}\right), t\right)\right)$ to the linear ODE $(3.3)$ since $b, B, k, c$ and $\xi$ are continuous and nonnegative. A regularization process gives us the existence of a weak solution $u \in \mathcal{C}^{1}\left(\mathbb{R}^{+}, L^{1}\left(\mathbb{R}^{+}\right)\right)$for $\bar{u} \in \mathcal{C}^{1}\left(\mathbb{R}^{+}, L^{1}\left(\mathbb{R}^{+}\right)\right)$and $u^{*}\left(., T_{n}\right) \in L^{1}\left(\mathbb{R}^{+}\right)$. Moreover, we have $u(x, t) \geq$ 0 , a.e.

Step 2. The solution $u$ is compactly supported. Let us assume $\operatorname{supp}\left(u^{*}\left(., T_{n}\right)\right) \subset[0, M+$ $\left.a_{\infty} T_{n}\right]$ and $\operatorname{supp}(\bar{u}(., t)) \subset\left[0, M+a_{\infty} t\right]$ for $t \geq T_{n}$ for some $M>x_{M}^{\xi}$. The characteristic curve passing through a point $(x, t)$ where $x>M+a_{\infty} t$ emanates from $\left(x, T_{n}\right)$ where $x>M+a_{\infty} T_{n}$ (or from $\left(0, t_{0}\right)$ for a given $\left.t_{0} \geq 0\right)$. On the characteristic curves emanating from the anchors $\left(x, T_{n}\right)$ where $x \geq M+a_{\infty} T_{n} \geq x_{M}^{\xi}$, the solution $u$ is constantly equal to zero (both the initial condition and source term are zero) . Then, for $x>M+a_{\infty} t$, with $M>x_{M}^{\xi}$, we have $u(x, t)=0$.

\section{Step 3. The map $\mathbf{F}$ is a contraction.}

We prove here that $\mathbf{F}$ is a contractive map in the Banach space

$$
X=C^{+}\left(\left[T_{n}, T_{n}+T\right] ; L^{1}\left(\mathbb{R}^{+}, d x\right)\right), \quad\|u\|_{X}=\sup _{T_{n} \leq t \leq T_{n}+T}\|u(t, .)\|_{L^{1}\left(\mathbb{R}^{+}, d x\right)} .
$$

The proof is a straightforward adaptation from [17], page 59. For $\left(\overline{u_{1}}, \overline{u_{2}}\right) \in X^{2}$, we define $\left(u_{1}, u_{2}\right) \in$ $X^{2}$ as $u_{1}:=\mathbf{F}\left[\bar{u}_{1}\right]$ and $u_{2}:=\mathbf{F}\left[\bar{u}_{2}\right]$. The function $u=u_{1}-u_{2}$ then satisfies for $\bar{u}=\overline{u_{1}}-\overline{u_{2}}$

$$
\frac{\partial u(x, t)}{\partial t}+a(t) \frac{\partial u(x, t)}{\partial x}=b(t)\left(-B(x) u(x, t)+\int_{x}^{\infty} k(y, x) \bar{u}(y, t) d y\right) .
$$


We multiply the above equation by $\operatorname{sign}(u)$, integrate over $\mathbb{R}^{+}$, and intergrate with time to get

$$
\|u\|_{X} \leq T b_{\infty} B_{\infty}\|\bar{u}\|_{X},
$$

which means that as soon as $T<1 /\left(2 b_{\infty} B_{\infty}\right), \mathbf{F}$ is a strict contraction in the Banach space $X$ and this proves the existence of a unique fixed point. We can iterate the operator on $\left[T_{n}+T, T_{n}+\right.$ $2 T],\left[T_{n}+2 T, T_{n}+3 T\right], \ldots$ since the condition on $T$ does not depend on the iteration. With this iteration process, we have built a solution to $(26)$ in $\mathcal{C}^{+}\left(\left[T_{n},+\infty\right), L^{1}\left(\mathbb{R}^{+}\right)\right)$. Properties 1 and 2 of lemma 1 are preserved by the map $\mathbf{F}$ and are thus true for the fixed point.

The following sequence of Lemmas shows that the map $\mathbf{G}$ is a contraction from $Y$ to $Y$.

Lemma 2 ( $L^{1}$-estimate). Assume $a, b, c \in L^{\infty}\left(\mathbb{R}^{+}\right)$. The solution to 25 satisfies for $A_{1}=c_{\infty} I_{\xi}$

$$
\int_{\mathbb{R}^{+}} u^{*}\left(x, T_{n}\right) d x \leq A_{1} T_{n} \quad \text { implies } \quad \int_{\mathbb{R}^{+}} u(x, t) d x \leq A_{1} t, \quad t \geq T_{n} .
$$

Proof. We integrate equation (21) over $\mathbb{R}^{+}$and use the condition at $x=0$ and the compact support property

$$
\frac{d}{d t} \int_{\mathbb{R}^{+}} u(x, t) d x=c(t) I_{\xi} \leq c_{\infty} I_{\xi}, \quad t \geq T_{n},
$$

which directly implies Lemma 2 after a time integration.

Lemma 3 ( $W^{1,1}$ estimate). Assume $a, b, c \in L^{\infty}\left(\mathbb{R}^{+}\right)$. Then there are some positive constants $A_{2}$ and $A_{3}$ such that if $u^{*}$ satisfies the premise of Lemma 2, then the solution to 25] satisfies for $t \geq T_{n}$

$$
\int_{\mathbb{R}^{+}}\left|\frac{\partial u^{*}}{\partial x}\right|\left(x, T_{n}\right) d x \leq A_{2} T_{n}^{2}+A_{3} T_{n} \quad \text { implies } \quad \int_{\mathbb{R}^{+}}\left|\frac{\partial u}{\partial x}\right|(x, t) d x \leq A_{2} t^{2}+A_{3} t,
$$

with

$$
A_{2}=\frac{1}{2} b_{\infty} c_{\infty} I_{\xi}\left(M_{2}+M_{3}\right), \quad A_{3}=\left(x_{M}^{\xi}-x_{m}^{\xi}\right) c_{\infty} \xi_{\infty}^{\prime} .
$$

Proof. Let us first differentiate equation 25) with respect to $x$ (on a regularized solution):

$$
\begin{aligned}
\frac{\partial}{\partial t} \frac{\partial u}{\partial x}(x, t)+a(t) \frac{\partial}{\partial x} \frac{\partial u}{\partial x}(x, t)= & b(t)\left(-u(x, t) \frac{\partial}{\partial x} \int_{0}^{x} k(x, y) d y-B(x) \frac{\partial u}{\partial x}(x, t)\right. \\
& \left.+\frac{\partial}{\partial x} \int_{x}^{\infty} k(y, x) u(y, t) d y\right)+c(t) \frac{\partial \xi}{\partial x}(x) .
\end{aligned}
$$

We notice that, thanks to 18 (19)

$$
\frac{\partial}{\partial x} \int_{0}^{x} k(x, y) d y=\int_{0}^{x} \partial_{1} k(y, x) d y, \quad \frac{\partial}{\partial x} \int_{x}^{\infty} k(y, x) u(y, t) d y=\int_{x}^{\infty} \partial_{2} k(y, x) u(y, t) d y
$$

since $k(x, x)=0$. We now formally multiply (27) by $\operatorname{sign}\left(\frac{\partial u}{\partial x}(x, t)\right)$ (actually, we multiply by a regularization of $\operatorname{sign}\left(\frac{\partial u}{\partial x}(x, t)\right)$ and pass to the limit - see [18] for details). From this, we get

$$
\begin{aligned}
& \frac{\partial}{\partial t}\left|\frac{\partial u}{\partial x}\right|(x, t)+a(t) \frac{\partial}{\partial x}\left|\frac{\partial u}{\partial x}\right|(x, t)=b(t)\left(-B(x)\left|\frac{\partial u}{\partial x}(x, t)\right|-\operatorname{sign}\left(\frac{\partial u}{\partial x}(x, t)\right) u(x, t) \int_{0}^{x} \partial_{1} k(y, x) d y\right. \\
& \left.+\int_{x}^{\infty} \operatorname{sign}\left(\frac{\partial u}{\partial x}(x, t)\right) \partial_{2} k(y, x) u(y, t) d y\right)+c(t) \operatorname{sign}\left(\frac{\partial u}{\partial x}(x, t)\right) \frac{\partial \xi}{\partial x}(x)
\end{aligned}
$$


And, after integration we have

$$
\frac{d}{d t} \int_{\mathbb{R}^{+}}\left|\frac{\partial u}{\partial x}(x, t)\right| d x \leq F_{1}(t)+F_{2}(t)+F_{3}(t)+F_{4}(t)+F_{5}(t),
$$

where, using Lemma 2 for $F_{3}$ and $F_{5}$,

$$
\begin{gathered}
F_{2}(t)=-b(t) \int_{\mathbb{R}^{+}} B(x)\left|\frac{\partial u}{\partial x}(x, t)\right| d x \leq 0, \\
F_{3}(t) \leq b(t) \int_{\mathbb{R}^{+}} \int_{0}^{x}\left|\partial_{1} k(y, x)\right||u(x, t)| d y d x \leq b_{\infty} M_{2} c_{\infty} I_{\xi} t, \\
F_{5}(t) \leq b(t) \int_{\mathbb{R}^{+}} \int_{x}^{\infty}\left|\partial_{2} k(y, x)\right||u(y, t)| d y d x \leq b_{\infty} c_{\infty} I_{\xi} M_{3} t \\
F_{6}(t)=c(t) \int_{\mathbb{R}^{+}}\left|\frac{\partial \xi}{\partial x}(x)\right| d x \leq c_{\infty}\left(x_{M}^{\xi}-x_{m}^{\xi}\right) \xi_{\infty}^{\prime},
\end{gathered}
$$

and since $u(0, t)=0$, writing equation 25 at $x=0$ gives us

$$
F_{1}(t)=-a(t)\left|\frac{\partial u}{\partial x}(0, t)\right|=b(t) \int_{0}^{\infty} k(y, 0) u(y, t) d y+c(t) \xi(0)=0 .
$$

In summary, we have

$$
\frac{d}{d t} \int_{\mathbb{R}^{+}}\left|\frac{\partial u}{\partial x}(x, t)\right| d x \leq b_{\infty} c_{\infty} I_{\xi}(M 2+M 3) t+c_{\infty}\left(x_{M}^{\xi}-x_{m}^{\xi}\right) \xi_{\infty}^{\prime},
$$

Then, integrating over $\left[T_{n}, t\right]$ we obtain the conclusion of Lemma 3 .

Lemma 4 ( $L^{1}$-stability). For $\left(a_{1}, b_{1}, c_{1}\right)$ and $\left(a_{2}, b_{2}, c_{2}\right)$ two triplets of functions of $L^{\infty}\left(\mathbb{R}^{+}\right)$, if $u_{2}^{*}$ satisfies the premise of Lemma 2 and Lemma 3, the associated solutions $u_{1}$ and $u_{2}$ to (25) satisfy for $t \geq T_{n}$

$$
\int_{\mathbb{R}^{+}}|u(x, t)| d x \leq A_{4}\left\|a_{1}-a_{2}\right\|_{\infty} t^{3}+\left(A_{3}\left\|a_{1}-a_{2}\right\|_{\infty}+A_{5}\left\|b_{1}-b_{2}\right\|_{\infty}\right) t^{2}+I_{\xi}\left\|c_{1}-c_{2}\right\|_{\infty} t
$$

where

$$
u=u_{1}-u_{2}, \quad A_{4}=\frac{A_{2}}{3}, \quad A_{5}=2 B_{\infty} c_{\infty} I_{\xi} .
$$

Proof. The difference $u$ satisfies

$$
\begin{aligned}
\frac{\partial u}{\partial t}(x, t)=-a_{1}(t) \frac{\partial u}{\partial x}(x, t) & +\left(a_{2}(t)-a_{1}(t)\right) \frac{\partial u_{2}}{\partial x}(x, t) \\
& +b_{1}(t)\left(-B(x) u(x, t)+\int_{x}^{\infty} k(y, x) u(y, t) d y\right) \\
& +\left(b_{1}(t)-b_{2}(t)\right)\left(B(x) u_{2}(x, t)-\int_{x}^{\infty} k(y, x) u_{2}(y, t) d y\right) \\
& +\left(c_{1}(t)-c_{2}(t)\right) \xi(x) .
\end{aligned}
$$


Multiplying by $\operatorname{sign}(u(x, t))$ we obtain

$$
\begin{aligned}
\frac{\partial|u|}{\partial t}(x, t) \leq & -a_{1}(t) \frac{\partial|u|}{\partial x}(x, t)+\left|a_{2}(t)-a_{1}(t)\right|\left|\frac{\partial u_{2}}{\partial x}(x, t)\right| \\
& +b_{1}(t)\left(-B(x)|u(x, t)|+\int_{x}^{\infty} k(y, x)|u(y, t)| d y\right) \\
& +\left|b_{1}(t)-b_{2}(t)\right|\left(B(x)\left|u_{2}(x, t)\right|+\int_{x}^{\infty} k(y, x)\left|u_{2}(y, t)\right| d y\right) \\
& +\left|c_{1}(t)-c_{2}(t)\right| \xi(x) .
\end{aligned}
$$

Now, we integrate over $\mathbb{R}^{+}$and get

$$
\begin{aligned}
\frac{d}{d t} \int_{\mathbb{R}^{+}}|u(x, t)| d x \leq & \left|a_{2}(t)-a_{1}(t)\right| \int_{0}^{\infty}\left|\frac{\partial u_{2}}{\partial x}(x, t)\right| d x+\left|c_{1}(t)-c_{2}(t)\right| I_{\xi} \\
& +\left|b_{1}(t)-b_{2}(t)\right|\left(\int_{0}^{\infty} B(x)\left|u_{2}(x, t)\right| d x+\int_{0}^{\infty} \int_{x}^{\infty} k(y, x)\left|u_{2}(y, t)\right| d y d x\right),
\end{aligned}
$$

and so

$$
\frac{d}{d t} \int_{\mathbb{R}^{+}}|u(x, t)| d x \leq\left\|a_{1}-a_{2}\right\|_{\infty}\left(A_{2} t^{2}+A_{3} t\right)+I_{\xi}\left\|c_{1}-c_{2}\right\|_{\infty}+2\left\|b_{1}-b_{2}\right\|_{\infty} B_{\infty} c_{\infty} I_{\xi} t .
$$

Integrating over time and taking the supremum over $t \in\left[T_{n}, t\right]$, we arrive at the conclusion of Lemma 4 .

Lemma $5\left(L^{1}(x d x)\right.$ estimate - only for $\left.M_{1} \neq 0\right)$. Assume $a, b, c \in L^{\infty}\left(\mathbb{R}^{+}\right)$. Then, if $u^{*}$ satisfies the premise of Lemma 2, the solution u to 25 satisfies

$$
\int_{\mathbb{R}^{+}} u^{*}\left(x, T_{n}\right) x d x \leq p_{0} \quad \text { implies } \quad \int_{\mathbb{R}^{+}} u(x, t) x d x \leq A_{6} e^{A_{7}\left(t-T_{n}\right)}+A_{6}^{\prime} T_{n} e^{A_{7}\left(t-T_{n}\right)}, \quad t \geq T_{n}
$$

with

$$
A_{6}=p_{0}+\frac{c_{\infty}\left(b_{\infty} M_{1}+I_{\xi} a_{\infty}+b_{\infty} M_{0} I_{\xi}\right)}{b_{\infty}^{2} M_{1}^{2}}, \quad A_{6}^{\prime}=\frac{c_{\infty} I_{\xi}\left(b_{\infty} M_{0}+a_{\infty}\right)}{b_{\infty} M_{1}}, \quad A_{7}=b_{\infty} M_{1} .
$$

Proof. We multiply equation 25 by $x$ and integrate over $\mathbb{R}^{+}$to get

$$
\frac{d}{d t} \int_{R^{+}} x u(x, t) d x=a(t) \int_{\mathbb{R}^{+}} u(x, t) d x+c(t)+b(t) \int_{\mathbb{R}^{+}} \int_{0}^{y} k(y, x)(x-y) u(y, t) d x d y
$$

which implies

$$
\frac{d}{d t} \int_{\mathbb{R}^{+}} x u(x, t) d x \leq a_{\infty} c_{\infty} I_{\xi} t+c_{\infty}+b_{\infty}\left(M_{0} c_{\infty} I_{\xi} t+M_{1} \int_{\mathbb{R}^{+}} x u(x, t) d x\right) .
$$

The solutions to the ODE $g^{\prime}(t)=r+s t+w g(t)$ are written

$$
g_{C}(t)=C e^{w t}-\frac{r w+1}{w^{2}}-\frac{s}{w} t
$$

then, the solutions $f$ to

$$
f^{\prime}(t) \leq r+s t+w f(t), \quad f\left(T_{n}\right) \leq p_{0},
$$


satisfy for any $C \in \mathbb{R}$ (Gronwall Lemma)

$$
f(t) \leq g_{C}(t)+\left(f\left(T_{n}\right)-g_{C}\left(T_{n}\right)\right) e^{w\left(t-T_{n}\right)} .
$$

Thus, if we take $C$ such as $g_{C}\left(T_{n}\right)=p_{0}$, i.e. $C=\left(p_{0}+\frac{r w+s}{w^{2}}\right) e^{-w T_{n}}+\frac{s}{w} T_{n} e^{-w T_{n}}$ we have $f(t) \leq C e^{w t}$, which leads to Lemma 5 .

Lemma $6\left(W^{1,1}(x d x)\right.$ estimate - only for $\left.M_{1} \neq 0\right)$. Assume $a, b, c \in L^{\infty}\left(\mathbb{R}^{+}\right)$. If $u^{*}$ satisfies the premise of Lemma 2 and Lemma 5, and if

$$
\int_{\mathbb{R}^{+}}\left|\frac{\partial u^{*}}{\partial x}\left(x, T_{n}\right)\right| x d x \leq A_{9} T_{n}+A_{10} T_{n}^{2}+A_{11} T_{n}^{3},
$$

then the solution to 25 satisfies

$$
\int_{\mathbb{R}^{+}}\left|\frac{\partial u}{\partial x}(x, t)\right| x d x \leq A_{8} e^{A_{7}\left(t-T_{n}\right)}+A_{9} t+A_{10} t^{2}+A_{11} t^{3}+A_{12} T_{n} e^{A_{7}\left(t-T_{n}\right)}, \quad t \geq T_{n} .
$$

with

$$
A_{8}=\frac{b_{\infty} A_{6}\left(M_{2}+M_{3}\right)}{A_{7}}, A_{9}=c_{\infty}\left(x_{M}^{\xi}-x_{m}^{\xi}\right) x_{M}^{\xi} \xi_{\infty}^{\prime}, A_{10}=a_{\infty} \frac{A_{3}}{2}, A_{11}=a_{\infty} \frac{A_{2}}{3}, A_{12}=\beta_{\infty} A_{6}^{\prime} .
$$

Proof. Let us differentiate equation (25) with respect to $x$ and multiply the result by $x \operatorname{sign}\left(\frac{\partial u}{\partial x}(x, t)\right)$. This gives

$$
\begin{aligned}
\frac{\partial}{\partial t} x\left|\frac{\partial u}{\partial x}\right|(x, t) & +a(t) x \frac{\partial}{\partial x}\left|\frac{\partial u}{\partial x}\right|(x, t)=b(t)\left(-x B(x)\left|\frac{\partial u}{\partial x}(x, t)\right|-x \int_{0}^{x} \partial_{1} k(x, y) d y \operatorname{sign}\left(\frac{\partial u}{\partial x}(x, t)\right) u(x, t)\right. \\
& \left.+\operatorname{sign}\left(\frac{\partial u}{\partial x}(x, t)\right) x \int_{x}^{\infty} \partial_{2} k(y, x) u(y, t) d y\right)+c(t) \operatorname{sign}\left(\frac{\partial u}{\partial x}(x, t)\right) x \frac{\partial \xi}{\partial x}(x) .
\end{aligned}
$$

After integration and using the same kind of estimates as those used for Lemma 3, we have and using Lemma 5 .

$$
\frac{d}{d t} \int_{\mathbb{R}^{+}} x\left|\frac{\partial u}{\partial x}(x, t)\right| d x \leq b_{\infty} A_{6}\left(M_{2}+M_{3}\right) e^{A_{7} t}+a_{\infty}\left(A_{2} t^{2}+A_{3} t\right)+c_{\infty}\left(x_{M}^{\xi}-x_{m}^{\xi}\right) x_{M}^{\xi} \xi_{\infty}^{\prime}
$$

and the conclusion of Lemma 6 follows after time integration.

Lemma $7\left(L^{1}(x d x)\right.$-stability- only for $\left.M_{1} \neq 0\right)$. For $\left(a_{1}, b_{1}, c_{1}\right)$ and $\left(a_{2}, b_{2}, c_{2}\right)$ two triplets of functions of $L^{\infty}\left(\mathbb{R}^{+}\right)$, the associated solutions $u_{1}$ and $u_{2}$ to 25 satisfy

$$
\int_{\mathbb{R}^{+}} x|u(x, t)| d x \leq A_{13} e^{A_{7}\left(t-T_{n}\right)}+A_{14} t, \quad t \geq T_{n}
$$

where

$$
\begin{aligned}
A_{13} & =\frac{1}{A_{7}}+\frac{v}{A_{7}^{2}}+\frac{2 y}{A_{7}^{3}}+\frac{6 z}{A_{7}^{4}}, \quad A_{14}=\left(M_{1}+2 B_{\infty}\right)\left\|b_{1}-b_{2}\right\|_{\infty} A_{6}, \\
v & =\left(A_{3}\left\|a_{1}-a_{2}\right\|_{\infty}+M_{0} I_{\xi} c_{\infty}\left\|b_{1}-b_{2}\right\|_{\infty}+M_{0} I_{\xi} b_{\infty}\left\|c_{1}-c_{2}\right\|_{\infty}\right), \\
y & =M_{0}\left(\left(A_{2}+A_{3}\right)\left\|a_{1}-a_{2}\right\|_{\infty}+A_{5}\left\|b_{1}-b_{2}\right\|_{\infty}\right), \\
z & =M_{0} A_{4}\left\|a_{1}-a_{2}\right\|_{\infty} .
\end{aligned}
$$


Proof. We multiply the equation (3.3) (satisfied by $u$ ) by $x \operatorname{sign}(u(x, t))$ and we integrate it over $\mathbb{R}^{+}$ so that

$$
\begin{aligned}
& \frac{d}{d t} \int_{\mathbb{R}^{+}} x|u(x, t)| d x \leq-a_{1}(t) \int_{\mathbb{R}^{+}} x \frac{\partial|u|}{\partial x}(x, t) d x+\left|a_{1}(t)-a_{2}(t)\right| \int_{\mathbb{R}^{+}} x\left|\frac{\partial u_{2}}{\partial x}(x, t)\right| d x \\
& \quad+b_{1}(t) \int_{\mathbb{R}^{+}} \int_{0}^{x}(x-y) k(x, y)|u(x, t)| d x+\left|c_{1}(t)-c_{2}(t)\right| \int_{\mathbb{R}^{+}} x \xi(x) d x \\
& \quad+\left(b_{1}(t)-b_{2}(t)\right) \int_{\mathbb{R}^{+}} \int_{0}^{x} k(x, y)(x \operatorname{sign}(u(x, t))-y \operatorname{sign}(u(y, t))) u_{2}(x, t) d x .
\end{aligned}
$$

Then,

$$
\begin{aligned}
\frac{d}{d t} \int_{\mathbb{R}^{+}} x|u(x, t)| & d x \leq \underbrace{b_{\infty} M_{1}}_{A_{7}} \int_{\mathbb{R}^{+}} x|u(x, t)| d x+\left\|c_{1}-c_{2}\right\|_{\infty}+t^{3} M_{0} A_{4}\left\|a_{1}-a_{2}\right\|_{\infty} \\
& +t^{2} M_{0}\left(\left(A_{2}+A_{3}\right)\left\|a_{1}-a_{2}\right\|_{\infty}+A_{5}\left\|b_{1}-b_{2}\right\|_{\infty}\right) \\
& +t\left(A_{3}\left\|a_{1}-a_{2}\right\|_{\infty}+M_{0} I_{\xi} c_{\infty}\left\|b_{1}-b_{2}\right\|_{\infty}+M_{0} I_{\xi} b_{\infty}\left\|c_{1}-c_{2}\right\|_{\infty}\right) \\
& +\left(M_{1}+2 B_{\infty}\right)\left\|b_{1}-b_{2}\right\|_{\infty} A_{6} e^{A_{7} t}, \quad t \in\left[T_{n}, T_{n}+T\right] .
\end{aligned}
$$

A function satisfying $f^{\prime}(t) \leq u+v t+y t^{2}+z t^{3}+m e^{w\left(t-T_{n}\right)}+m^{\prime} T_{n} e^{w\left(t-T_{n}\right)}+w f(t)$ with $f\left(T_{n}\right)=0$ satisfies $f(t) \leq\left(\frac{1}{w}+\frac{v}{w^{2}}+\frac{2 y}{w^{3}}+\frac{6 z}{w^{4}}\right) e^{w\left(t-T_{n}\right)}+m t$, and we obtain Lemma 7 .

Lemma 8 (A-priori estimate for the solution). Let us assume that there exists a solution $(u, p) \in$ $C\left(\mathbb{R}^{+} ; L^{1}\left(\mathbb{R}^{+},(1+x) d x\right)\right) \times C\left(\mathbb{R}^{+}\right)$to the system (10), (11) with boundary conditions (12) for $t \in$ $\left[0, T_{n}\right]$ for some given $T_{n} \geq 0$. Then, it satisfies

$$
\begin{gathered}
0 \leq p(t) \leq p_{0}, \quad \int_{\mathbb{R}^{+}} u(x, T) x d x \leq p_{0}, \quad \text { and } \quad \int_{\mathbb{R}^{+}} u(x, T) d x \leq N\left(p_{0}\right) I_{\xi} T, \quad \text { for } T \geq 0 . \\
\text { and } \quad \int_{\mathbb{R}^{+}}\left|\frac{\partial u}{\partial x}(x, T)\right| d x \leq K_{1} T+K_{2} T^{2}, \quad \text { for } \quad T>0,
\end{gathered}
$$

for some positive constants $K_{1}$ and $K_{2}$ given by

$$
K_{1}=\left(x_{M}^{\xi}-x_{m}^{\xi}\right) N\left(p_{0}\right) \xi_{\infty}^{\prime}, \quad K_{2}=\frac{\beta\left(p_{0}\right)}{2} N\left(p_{0}\right) I_{\xi}\left(M_{2}+M_{3}\right) .
$$

Proof. The proof of (29) is a direct application of Lemma 3.

\subsection{The map $\mathrm{G}$ is a contraction}

We now show that $\mathbf{G}$ is a contractive map.

Lemma 9 ( Estimate for $\|p\|_{Y}$ ). We fix $T_{n} \geq 0$. For a couple of functions $\left(\overline{p_{1}}, \overline{p_{2}}\right) \in Y^{2}$, we define $p_{1}=\mathbf{G}\left[\overline{p_{1}}\right], p_{2}=\mathbf{G}\left[\overline{p_{2}}\right], \bar{p}:=\bar{p}_{1}-\bar{p}_{2}$ and $p:=p_{1}-p_{2}$, where the initial conditions $p_{1}^{*}, p_{2}^{*}, u_{1}^{*}$ and $u_{2}^{*}$ are solutions to (10), (11), (12) over $\left[0, T_{n}\right]$. (We recall that $Y$ and $\mathbf{G}$ depend on $T_{n}$.) Then we have

1. In the case $M_{1}=0$,

$$
\|p\|_{Y} \leq T\left(A_{15}\left(T_{n}+T\right)^{3}+A_{16}\left(T_{n}+T\right)^{2}+A_{17}\left(T_{n}+T\right)+A_{18}\right)\|\bar{p}\|_{Y} .
$$


2. In the case $M_{1} \neq 0$,

$$
\|p\|_{Y} \leq T\left(A_{15}\left(T_{n}+T\right)^{3}+A_{16}\left(T_{n}+T\right)^{2}+\tilde{A_{17}}\left(T_{n}+T\right)+A_{18}+A_{19} e^{A_{7}\left(T_{n}+T\right)}\right)\|\bar{p}\|_{Y},
$$

where

$$
\begin{aligned}
& A_{15}=\left(\beta_{\infty} M_{0}+\gamma_{\infty}\right) A_{4} \alpha, \quad A_{16}=\left(\beta_{\infty} M_{0}+\gamma_{\infty}\right)\left(A_{3} \alpha+A_{5} \beta_{\infty}^{\prime}\right), \\
& A_{17}=N_{\infty}^{\prime} I_{\xi}\left(\beta_{\infty} M_{0}+\gamma_{\infty}\right)+N_{\infty} I_{\xi}\left(\alpha+\beta_{\infty}^{\prime} M_{0}\right), \\
& \tilde{A_{17}}=A_{17}+M_{1} \beta_{\infty} A_{12}, \quad A_{18}=N_{\infty}^{\prime}, \quad A_{19}=M_{1}\left(\beta_{\infty}^{\prime} A_{6}+\beta_{\infty} A_{13}\right), \quad \tilde{A_{19}}=M_{1} \beta_{\infty}^{\prime} A_{6}^{\prime} .
\end{aligned}
$$

Proof. We denote by $u_{i}$ the unique solution of (21) with $\bar{p}=\bar{p}_{i}$. We define $u:=u_{1}-u_{2}$. We have

$$
\begin{aligned}
\frac{d p}{d t}(t)= & -\gamma\left(\overline{p_{1}}(t)\right) \int_{\mathbb{R}^{+}} u(x, t) d x-\left(\gamma\left(\overline{p_{1}}(t)\right)-\gamma\left(\overline{p_{2}}(t)\right)\right) \int_{\mathbb{R}^{+}} u_{2}(x, t) d x \\
& +\beta\left(\overline{p_{2}}(t)\right) \int_{\mathbb{R}^{+}} u(y, t)\left(\int_{0}^{y}(y-x) k(y, x) d x\right) d y \\
& +\left(\beta\left(\overline{p_{1}}(t)\right)-\beta\left(\overline{p_{2}}(t)\right)\right) \int_{\mathbb{R}^{+}} u_{1}(y, t)\left(\int_{0}^{y}(y-x) k(y, x) d x\right) d y \\
& +N\left(\overline{p_{1}}(t)\right)-N\left(\overline{p_{2}}(t)\right), \quad t \in\left[T_{n}, T_{n}+T\right]
\end{aligned}
$$

which after integration over $\left[T_{n}, t\right]$ for $t \in\left[T_{n}, T_{n}+T\right]$ gives us

$$
\begin{aligned}
\left|p_{1}(t)-p_{2}(t)\right| & \leq \int_{T_{n}}^{t} \gamma\left(\overline{p_{1}}(s)\right) \int_{\mathbb{R}^{+}}|u(x, s)| d x d s+\alpha \int_{T_{n}}^{t}\left|\overline{p_{1}}(s)-\overline{p_{2}}(s)\right| \int_{\mathbb{R}^{+}} u_{2}(x, s) d x d s \\
& +\beta_{\infty} \int_{T_{n}}^{t} \int_{\mathbb{R}^{+}}|u(y, s)|\left(\int_{0}^{y}(y-x) k(y, x) d x\right) d y \\
& \left.+\beta_{\infty}^{\prime} \int_{T_{n}}^{t} \mid \overline{p_{1}}(s)\right)-\overline{p_{2}}(s)\left|\int_{\mathbb{R}^{+}}\right| u_{2}(y, s) \mid\left(\int_{0}^{y}(y-x) k(y, x) d x\right) d y d s \\
& +N_{\infty}^{\prime} \int_{T_{n}}^{t}\left|\overline{p_{1}}(s)-\overline{p_{2}}(s)\right| d s, \quad t \in\left[T_{n}, T_{n}+T\right] .
\end{aligned}
$$

We take the supremum over $\left[T_{n}, T_{n}+T\right]$ on both sides such that

$$
\begin{aligned}
\|p\|_{Y} & \leq T\|\bar{p}\|_{Y}\left(N_{\infty}^{\prime}+\int_{\mathbb{R}^{+}}\left(\beta_{\infty}^{\prime}\left(M_{0}+M_{1} x\right)+\alpha\right) \sup _{t \in\left[T_{n}, T_{n}+T\right]}\left|u_{2}(x, t)\right| d x\right) \\
& +T\left(\int_{\mathbb{R}^{+}}\left(\gamma_{\infty}+\beta_{\infty}\left(M_{0}+M_{1} x\right)\right) \sup _{t \in\left[T_{n}, T_{n}+T\right]}|u(x, t)| d x\right) .
\end{aligned}
$$

We now use the previous Lemmas that we apply for $a_{\infty}=\gamma_{\infty}, b_{\infty}=\beta_{\infty}, c_{\infty}=N_{\infty},\left\|a_{1}-a_{2}\right\|_{\infty}=\alpha\|\bar{p}\|_{Y},\left\|b_{1}-b_{2}\right\|_{\infty}=\beta_{\infty}^{\prime}\|\bar{p}\|_{Y},\left\|c_{1}-c_{2}\right\|_{\infty}=N_{\infty}^{\prime}\|\bar{p}\|_{Y}$.

We distinguish two cases. First, we consider $M_{1}=0$. Using Lemma (8), we have

$$
\int_{\mathbb{R}^{+}} u_{2}\left(x, T_{n}\right) d x=\int_{\mathbb{R}^{+}} u_{2}^{*}\left(x, T_{n}\right) d x \leq N\left(p_{0}\right) I_{\xi} T_{n},
$$

so that Lemma (2) implies

$$
\int_{\mathbb{R}^{+}} u_{2}(x, t) d x \leq N_{\infty} I_{\xi}\left(T_{n}+T\right), \quad t \in\left[T_{n}, T_{n}+T\right] .
$$


Since

$$
\int_{\mathbb{R}^{+}}\left|\frac{\partial u_{2}}{\partial x}\left(x, T_{n}\right)\right| d x=\int_{\mathbb{R}^{+}}\left|\frac{\partial u_{2}^{*}}{\partial x}\left(x, T_{n}\right)\right| d x \leq K_{1} T_{n}+K_{2} T_{n}^{2} \leq A_{2} T_{n}^{2}+A_{3} T_{n},
$$

we can apply Lemma 4 and obtain

$$
\begin{aligned}
\|p\|_{Y} & \leq T\|\bar{p}\|_{Y}\left(N_{\infty}^{\prime}+\left(T_{n}+T\right)\left(N_{\infty}^{\prime} I_{\xi}\left(\beta_{\infty} M_{0}+\gamma_{\infty}\right)+N_{\infty} I_{\xi}\left(\alpha+\beta_{\infty}^{\prime} M_{0}\right)\right)\right. \\
& \left.+\left(T_{n}+T\right)^{2}\left(\beta_{\infty} M_{0}+\gamma_{\infty}\right)\left(A_{3} \alpha+A_{5} \beta_{\infty}^{\prime}\right)+\left(T_{n}+T\right)^{3}\left(\beta_{\infty} M_{0}+\gamma_{\infty}\right) A_{4} \alpha\right)
\end{aligned}
$$

which leads to the conclusion of the first item of Lemma 9. For the case $M_{1} \neq 0$, Lemma 8 implies that

$$
\int_{\mathbb{R}^{+}} x u\left(x, T_{n}\right) d x \leq p_{0}
$$

so that we can apply Lemma 5. We also use Lemma 7 and we obtain

$$
\begin{aligned}
\|p\|_{Y} & \leq T\|\bar{p}\|_{Y}\left(N_{\infty}^{\prime}+\left(T_{n}+T\right)\left(N_{\infty}^{\prime} I_{\xi}\left(\beta_{\infty} M_{0}+\gamma_{\infty}\right)+N_{\infty} I_{\xi}\left(\alpha+\beta_{\infty}^{\prime} M_{0}\right)+\beta_{\infty} M_{1} A_{14}\right)\right. \\
& +\left(T_{n}+T\right)^{2}\left(\beta_{\infty} M_{0}+\gamma_{\infty}\right)\left(A_{3} \alpha+A_{5} \beta_{\infty}^{\prime}\right)+\left(T_{n}+T\right)^{3}\left(\beta_{\infty} M_{0}+\gamma_{\infty}\right) A_{4} \alpha \\
& \left.+M_{1}\left(\beta_{\infty}^{\prime} M_{1}\left(A_{6}+A_{6}^{\prime} T_{n}\right)+\beta_{\infty} A_{13}\right) e^{A_{7} T}\right)
\end{aligned}
$$

which ends the proof of Lemma 9 .

The next Lemma is the last Lemma required for the proof of Theorem 1 .

Lemma 10. We can build a sequence $T_{0}=0, T_{1}, \ldots, T_{n}, T_{n+1}, \ldots$ such that

1. For all $n \in \mathbb{N}$, there exists a unique solution to system (10) for $t \in\left[0, T_{n}\right]$,

2. $\lim _{n \rightarrow \infty} T_{n}=+\infty$.

Proof. Let us assume that $\left(u^{*}, p^{*}\right)$ is a solution to system (10) (11) for $t \in\left[0, T_{n}\right]$. For the couple of functions $\left(\overline{p_{1}}, \overline{p_{2}}\right) \in Y^{2}$, we define $p_{1}=\mathbf{G}\left[\overline{p_{1}}\right]$ and $p_{2}=\mathbf{G}\left[\overline{p_{2}}\right]$. Lemma 9 gives us

$$
\begin{aligned}
\left\|\mathbf{G}\left[\overline{p_{1}}\right]-\mathbf{G}\left[\overline{p_{2}}\right]\right\|_{Y} \leq T\left(A_{15}\left(T_{n}+T\right)^{3}\right. & +A_{16}\left(T_{n}+T\right)^{2}+\tilde{A_{17}}\left(T_{n}+T\right)+A_{18}+A_{19} e^{A_{7} T} \\
& \left.+\tilde{A}_{19} T_{n} e^{A_{7} T}\right)\left\|\overline{p_{1}}-\overline{p_{2}}\right\|_{Y}
\end{aligned}
$$

where the $A_{i}^{\prime} s$ are those defined in Lemma 9. The map $\mathbf{G}$ is a strict contraction provided that the sum in (30) is strictly less than 1, which is implied by the fact that each of the 6 terms is strictly less than $1 / 6$. As a consequence, the map $\mathbf{G}$ is a strict contraction over $\left[T_{n}, T_{n}+T\right]$ as soon as $T$ satsifies the 5 following conditions:

$$
\begin{aligned}
& T<\frac{1}{6 A_{18}}=: G_{1}\left(T_{n}\right), T<\frac{1}{6 \tilde{A}_{17}\left(T_{n}+\frac{1}{6 A_{18}}\right)}=: G_{2}\left(T_{n}\right), T<\frac{1}{6 A_{16}\left(T_{n}+\frac{1}{6 A_{18}}\right)^{2}}=: G_{3}\left(T_{n}\right), \\
& T<\frac{1}{6 A_{15}\left(T_{n}+\frac{1}{6 A_{18}}\right)^{3}}=: G_{4}\left(T_{n}\right), T<\frac{1}{A_{19}} e^{-A_{7}\left(T_{n}+\frac{1}{6 A_{18}}\right)}=: G_{5}\left(T_{n}\right), \\
& T<\frac{1}{A_{19} T_{n}} e^{-A_{7}\left(T_{n}+\frac{1}{6 A_{18}}\right)}=: G_{6}\left(T_{n}\right) .
\end{aligned}
$$


Then, the Banach fixed point theorem guarantees that we can extend the solution to $\left[T_{n}, T_{n}+T\right]$. The sequence defined by induction through

$$
T_{0}=0, \quad T_{n+1}=T_{n}+\min \left\{G_{1}\left(T_{n}\right), G_{2}\left(T_{n}\right), G_{3}\left(T_{n}\right), G_{4}\left(T_{n}\right), G_{5}\left(T_{n}\right), G_{6}\left(T_{n}\right)\right\},
$$

is diverging, since it is strictly increasing and the application $x \rightarrow x+\min \left\{G_{1}(x)+G_{2}(x)+G_{3}(x)+\right.$ $\left.G_{4}(x)+G_{5}(x)+G_{6}(x)\right\}$ has no fixed point.

\section{Numerical results}

In this section, we describe results of the numerical simulation of equations (1), (2), and (3). First, we outline the numerical details of our approach. Then, we illustrate the behavior of our model by running a variety of simulations. In particular, we show how variations in certain model parameters change the qualitative and quantitative behavior of solutions. Such exploration gives us a better idea as to which parameters might be influenced by the addition of MTAs into a system of growing MTs. We simulate our model using a finite difference method. For the advection terms in equation (1) we use an upwinding approach, and an explicit Euler strategy for the ODEs (2) and (3). All integral terms are calculated using an order 0 quadrature method, adjusted to preserve tubulin at the discrete level.

All simulations are implemented in Matlab using custom code. We discretize our domain into 100 grid points, where the distance between each grid point, $\Delta x$, is constant and equal to $0.2 \mu \mathrm{m}$. Thus, our domain is $10 \mu \mathrm{m}$ in length. As we are only considering short time simulations, this domain size is appropriate (i.e., MTs do not grow larger than this size). Also, we choose an appropriate time step so that our scheme satisfies the CFL condition [25]. Our time step, $\Delta t$, equals 0.001 seconds.

\subsection{Parameter values in the base case}

Table 1: Table of model parameters

\begin{tabular}{|c|c|c|}
\hline Model parameter & Description & value \\
\hline$\alpha$ & growth parameter & $2 \frac{\mu \mathrm{m}}{\mathrm{M} \cdot \mathrm{min}}$ \\
\hline$\gamma^{h}$ & Baseline hydrolysis rate & $5 \frac{\mu \mathrm{m}}{\mathrm{min}}$ \\
\hline$\beta_{\infty}$ & Shortening rate & $20 \frac{\mu \mathrm{m}}{\mathrm{min}}$ \\
\hline$x_{0}$ & Shortening distance & $1.6 \mu \mathrm{m}$ \\
\hline$\sigma$ & Standard deviation of $x_{0}$ & $0.2 \mu \mathrm{m}$ \\
\hline$\mu$ & Nucleation rate & $0.1 \frac{1}{\mathrm{M} \cdot \mathrm{min}}$ \\
\hline$m$ & Dimer nucleation number & 2 \\
\hline$C_{N}$ & Normalization constant (from equation $(8)) C_{N}$ & $1 / 4$ \\
\hline$x_{\min }$ & Maximal size of nucleated MTs & $0.8 \mu \mathrm{m}$ \\
\hline$\kappa$ & GDP/GTP-tubulin exchange rate & $2 \frac{1}{\mathrm{~min}}$ \\
\hline$p_{c}$ & Critical growth value & $2 \mu \mathrm{M}$ \\
\hline$p_{N}$ & Critical nucleation value & $12 \mu \mathrm{M}$ \\
\hline$p_{0}$ & Initial GDP concentration & $15 \mu \mathrm{M}$ \\
\hline$p_{h}$ & Critical fragmentation value & $4.5 \mu \mathrm{M}$ \\
\hline
\end{tabular}

Our base case parameters for the numerical simulations are summarized in Table 1. For some model parameters, their order of magnitude was determined from the literature. In particular, the 
hydrolysis rate is found in [4], the shortening rate $\beta_{\infty}$ in [23], the dimer nucleation number $m$ in [19], and the GDP/GTP-tubulin exchange rate $\kappa$ in [23]. Other parameters were estimated through discussions with biologists [8]. The purpose of simulations is to provide insight into the qualitative behavior of our system for realistic model parameters, not to provide quantitative results.

\subsection{Simulation results in the base case}

We first describe simulation output that can be compared with experimental data.

One quantity of interest is the average MT length (i.e., the total amount of tubulin in polymer form). This average, between $T_{\min }$ and $T_{\max }$, is defined as

$$
\bar{u}_{t o t}=\frac{1}{T_{\max }-T_{\min }} \int_{T_{\min }}^{T_{\max }} u_{t o t}(t) d t, \quad u_{t o t}(t)=\int_{0}^{\infty} u(x, t) x d x,
$$

where $T_{\min }$ is the time at which the first GTP-tubulin population begins to stabilize (we will describe this in more detail later in this section), $T_{\max }$ is the maximum time of simulation, and $u_{\text {tot }}(t)$ is the total tubulin in polymerized form at time $t$.

Similarly, we can write the mean MT growth rate as

$$
\bar{\gamma}=\frac{1}{T_{\max }-T_{\min }} \int_{T_{\min }}^{T_{\max }} \gamma(p(t)) d t .
$$

This quantity can be estimated experimentally using data collected from kymographs (recall Figure 2).

In many of the existing models used to describe MT dynamic instability, information regarding MT growth and shortening, as well as the frequencies of switching between states of growing and shortening, are fixed parameters [3]. In particular, these parameters are required as model input for the simulation of MT dynamics. In experiments, these parameters are observables, and can vary depending on the conditions of the system. Our model is different from other models, as we only require information about how MTs shorten and grow. The frequency of switching between growing and shortening states, as well as the mean MT growth rate, cannot be determined exactly from the outputs of our model. However, we suggest a way to roughly estimate these quantities. The frequency of switching between growth and shortening is referred to as the catastrophe frequency $\left(f_{c}\right)$, while the frequency of switching between shortening and growth is referred to as the rescue frequency $\left(f_{r}\right)$. Using the common definitions described in Walker et al. [23] we write,

$$
f_{c}=\frac{\text { number of catastrophe events }}{\text { total time spent growing }} \text { and } \quad f_{r}=\frac{\text { number of rescue events }}{\text { total time spent shrinking }} \text {. }
$$

We assume that at locations where the function $u_{t o t}(t)$ attains an extremum, a catastrophe or a rescue occurs. We define a sequence of times $T_{1}, T_{2}, \ldots . T_{K}$, as times at which maxima (catastrophe) and minima (rescues) occur, where $M$ denotes the total number of catastrophe events and $N$ the total number of rescue events $(M+N=K)$. MTs start growing from zero length at time $T_{0}$, thus $T_{1}$ necessarily corresponds to the time of the first catastrophe event. Further, since a catastrophe is always followed by a rescue (and vice versa), the following definitions precisely describe the catastrophe and rescue frequencies:

$$
f_{c}=\frac{M-1}{\sum_{i=1}^{M-1}\left[T_{2 i+1}-T_{2 i}\right]} \quad \text { and } \quad f_{r}=\frac{N}{\sum_{i=1}^{N}\left[T_{2 i}-T_{2 i-1}\right]} .
$$

Here, we do not include the first period of growth $\left(T_{1}-T_{0}\right)$ into our definition for total time spent growing, as it is during this time that freshly nucleated MTs are introduced into the system. 
A comparison between the prediction of our model in the base case and the outputs of experiments performed by the group of Honoré et al. [16] is displayed in Table 2, Note that for this particular experiment, there was no information for total polymer concentration $u_{t o t}$.

Table 2: Table of model output for $k_{0}$ compared with experiment

\begin{tabular}{llllll}
\hline & $f_{c}$ & $f_{r}$ & $\bar{u}_{t o t}$ & $\bar{\gamma}$ & Time spent shortening \\
Units & $\min ^{-1}$ & $\min ^{-1}$ & $\mu \mathrm{M}$ & $\frac{\mu \mathrm{m}}{\min }$ & percent \\
Model ouput & 2.38 & 9.17 & 7.9 & 5.2 & 28 \\
Experimental output & 2.57 & 7.12 & $\mathrm{~N} / \mathrm{A}$ & $12.176 \pm 7.8$ & 25.21 \\
\hline
\end{tabular}
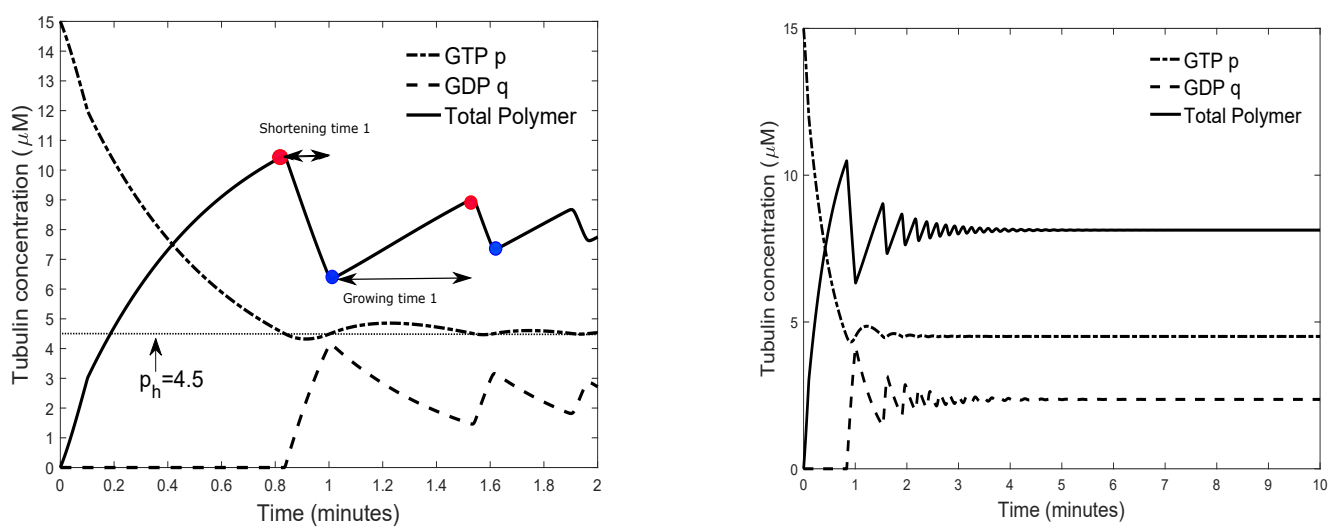

Figure 3: Left: Example of oscillating populations of tubulin found in MTs, free GTP-tubulin, and free GDPtubulin. Parameters used are summarized in Table 1 Shortening kernel $k_{0}$ is used. Right: Extended simulation of the figure to the left. MT dynamic instability is only sustained over a relatively short period of time. For large time, MT dynamics are completely suppressed.

Figures 3 (Left) and (Right) illustrate oscillating populations of tubulin in polymer form as well as oscillating populations of free GTP- and GDP-tubulin using the base case model parameters given in Table 1. In Figure 3 (Left), we highlight catastrophe and rescue events by red dots and blue dots, respectively. Also, examples of shortening and growing time periods are highlighted. Using Figure 3 (Left), we calculate values for $f_{c}$ and $f_{r}$ using formulas (31), and the mean MT length $\bar{u}_{\text {tot }}$ and growth rate $\bar{\gamma}$, using formulas $(4.2)$ and $(4.2)$, where $T_{\min }$ is set to $T_{1}$ (the time of the first catastrophe event). From Figure 3 (Left), we see that at $T_{1}$ the GTP-tubulin concentration approaches a mean value which is close in value to $p_{h}$.

Functions of the system (1) , (2), and (3) that depend on the value of $p$ include the nucleation rate $N(p)$, the shortening rate $\beta(p)$, and the growth rate $\gamma(p)$. Recall that, the critical nucleation value $p_{N}=12 \mu \mathrm{M}$, the critical growth value $p_{c}=2 \mu \mathrm{M}$ and the critical fragmentation value $p_{h}=$ $\frac{\gamma^{h}+\alpha p_{c}}{\alpha}=4.5 \mu \mathrm{M}$. At time $t=0, p=15 \mu \mathrm{M}$, thus $N(p)>0, \beta(p)=0$ and $\gamma(p)>0$ : new microtubules are formed by nucleation and they grow so that the total mass of microtubules increases and $p$ decreases. At time $t=0.1, p$ goes below the critical nucleation value $p_{N}$; from that point, nucleation stops, which slows down the creation of polymerized tubulin. At time $t=0.8, p$ reaches the critical value $p_{h}$ and fragmentation is initiated: microtubules start to shorten, and free GDPtubulin $(q)$ is created. We notice that the amplitude of the oscillations, as well as the period of oscillation, decrease with time. This result suggests that MT dynamic instability may only be sustained over a relatively short period of time. Figure 3 (right) illustrates the dynamics of MTs 
over a ten minute time period.

\subsection{Comparison between $k_{0}$ and $k_{1}$ : a closer look at shortening distance $x_{0}$ and shortening rate $\beta_{\infty}$}

In this section, we show simulation results for system (1), (2), and (3). For the different shortening kernels, $k_{0}$ and $k_{1}$, described in equation (6), we will compare long-term tubulin concentrations, as well as long-term MT length distributions.

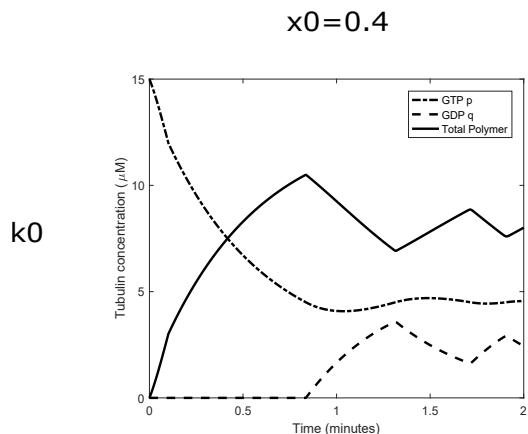

(a)

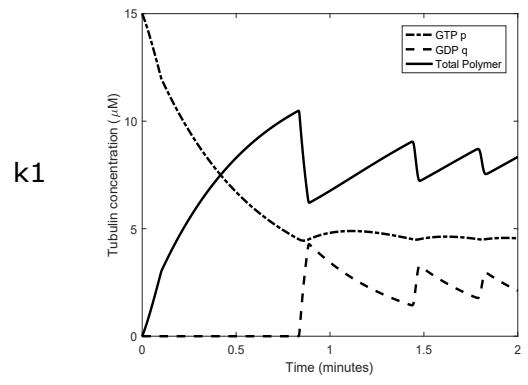

(d)

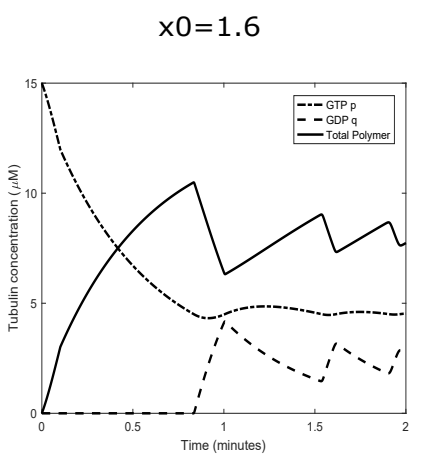

(b)

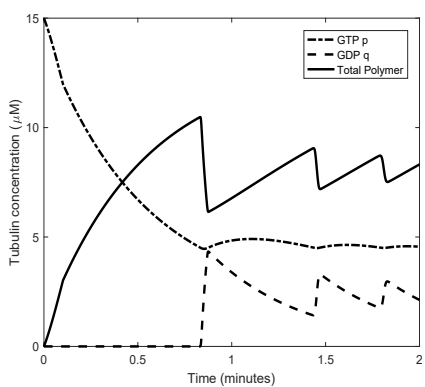

(e)

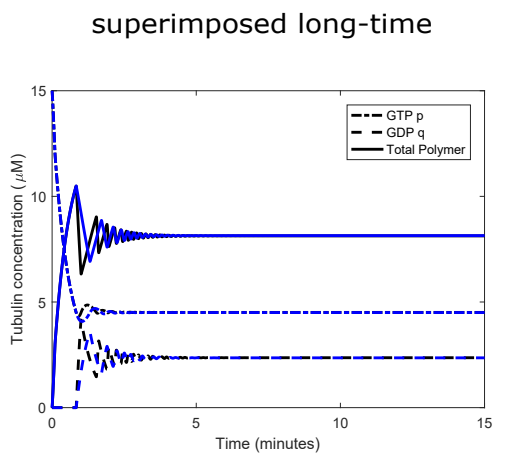

(c)

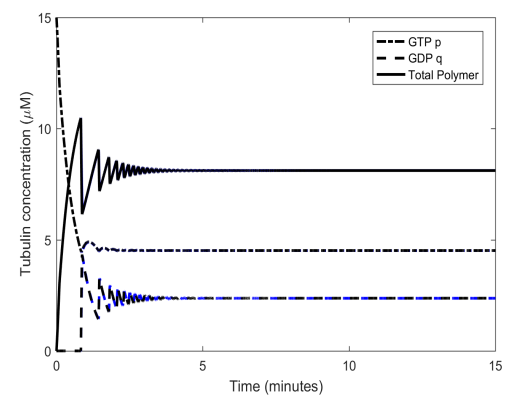

(f)

Figure 4: Examination of tubulin concentrations for varying $x_{0}$ and $k_{i}$.

Figures 4 and 5 , illustrate MT tubulin concentrations for varying shortening distance $x_{0}$ and varying shortening rate $\beta_{\infty}$, respectively. Here, we note that short-term dynamics differ for differing values of $x_{0}$ and $\beta_{\infty}$ for kernel $k_{0}$, but not for $k_{1}$. Further, we note the long-term dynamics (i.e., the steady-state values of GTP-tubulin, GDP-tubulin, and total tubulin in polymer form) remain the same in all cases, regardless of the shortening distance $x_{0}$, rate $\beta_{\infty}$, or kernel $k_{i}$ used (result shown in panels (c) and (f) of Figures 4 and 5 .) 


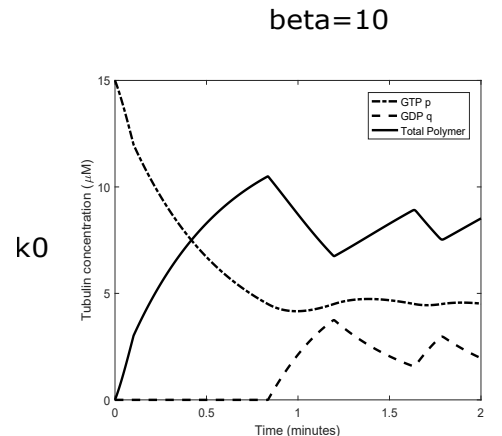

(a)

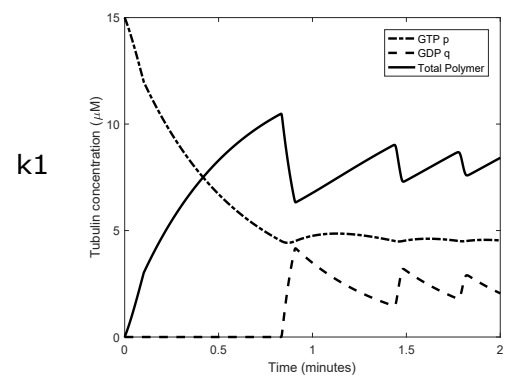

(d)

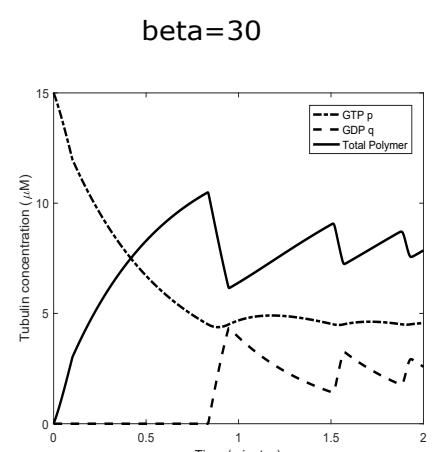

(b)

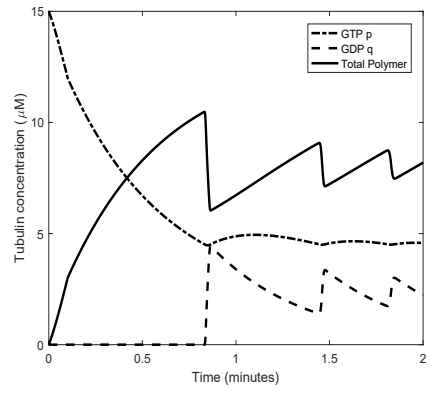

(e)

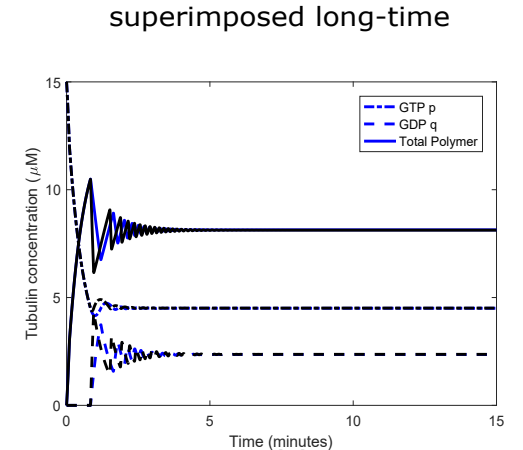

(c)

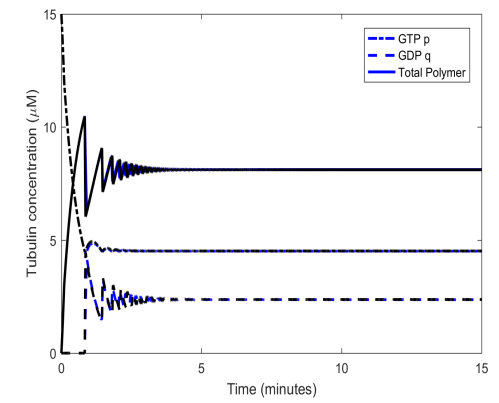

(f)

Figure 5: Examination of tubulin concentrations for varying $\beta_{\infty}$ and $k_{i}$.

Figures 6 and 7 show the temporal evolution of the MT length distributions for varying shortening distance $x_{0}$ and varying shortening rate $\beta_{\infty}$, respectively. Figures 6(c) and (f) illustrate the long-term (steady-state) MT length distributions. From these figures, we see that larger values of $x_{0}$, result in distributions with higher numbers of shorter MTs. This result makes sense, as MTs undergo larger shortening events for higher values of $x_{0}$. Unlike these results, Figures 7(c) and (f) illustrate that steady-state MT length distributions are very close for each $k_{i}$, regardless of the value of $\beta_{\infty}$ used.

\subsection{Dependence of $\gamma^{h}$ and $\kappa$ on MT dynamics: insight into drug effects}

In this section, we show how variations in certain model parameters change the total polymerized tubulin (MT length), and in some cases we also show how these parameter variations change the GTP-tubulin concentration. We do so, as this information will provide insight into which parameters may be altered by the introduction of stabilizing and destabilizing drugs. At low doses, destabilizing MTAs [26] promote catastrophe and reduce polymer mass, whereas at high doses, destabilizing MTAs [26] suppress MT dynamics, and MTs completely depolymerize. At almost all doses, stabilizing MTAs promote MT polymerization and stabilization, i.e., MT catastrophe is suppressed, and polymer tubulin concentration is increased.

Dependence to the hydrolysis rate $\gamma^{h}$. Figure 8 corresponds to simulation results for changes in the hydrolysis rate from the base case value (see Table 1 ). Here, we increase and decrease the critical fragmentation value, $p_{h}$, from it's base case value of 4.5 . This is equivalent to increasing/decreasing the hydrolysis rate $\gamma^{h}$.

From Figure 8 (left), we see a decrease in the mean polymer density $\bar{u}_{t o t}$ for increasing hydrolysis 


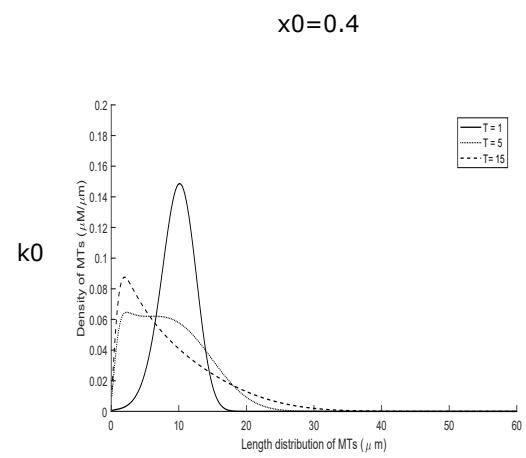

(a)

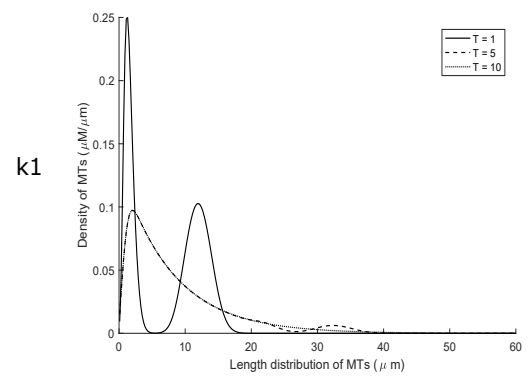

(d)

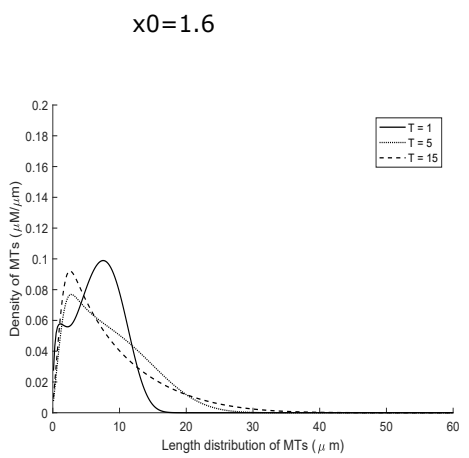

(b)

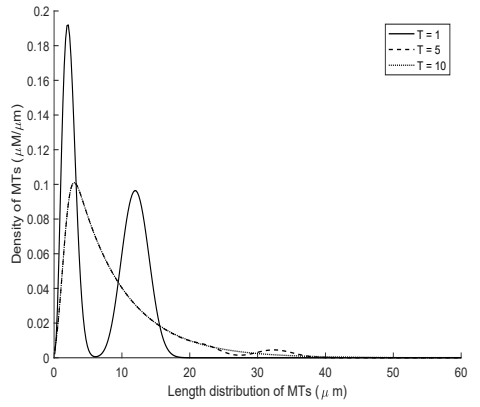

(e) superimposed long-time

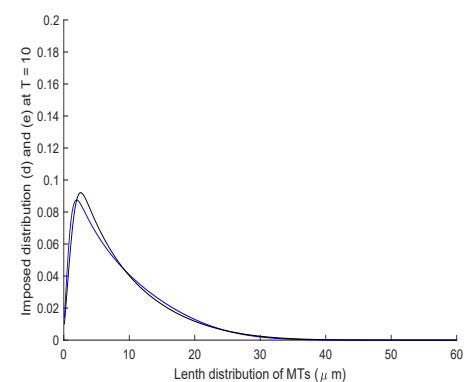

(c)

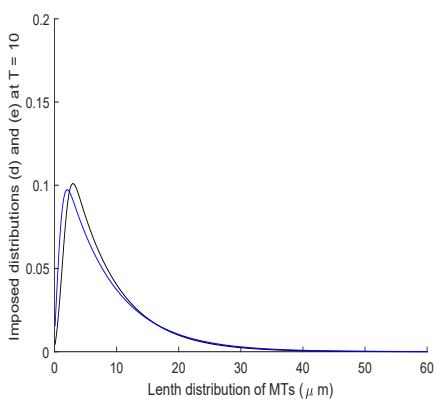

(f)

Figure 6: Examination of polymer length distribution for varying $x_{0}$ and $k_{i}$. In (c) and (f) the blue curves correspond to $x_{0}=0.4 \mu \mathrm{m}$. For each $k_{i}$, the long-time length distribution is different, depending on the value of $x_{0}$.

rate. Further, we see that decreases in the hydrolysis rate (from the base case) work to suppress MT dynamics by reducing the number of catastrophe events. These results are consistent with the action of MT destabilizing (vinblastine or vincristine [26]) and stabilizing (taxol [26]) MT targeting agents (MTAs), respectively. Thus, we suggest that MT destabilizing MTAs may regulate MT dynamics by increasing the hydrolysis rate, and that MT stabilizing MTAs may work to increase MT polymerization through reduction of the hydrolysis rate.

Figure 8(right) shows the corresponding changes in the GTP-tubulin concentration. Here, it is noted that as the hydrolysis rate $\gamma^{h}$ is increased, the level of free GTP-tubulin in the system is increased. This result makes sense, due to the fact that as a MT depolymerizes (at high values of the hydrolysis rate), free tubulin is released back into the system.

Dependence to the recycling rate $\kappa$. Next, we show results for variations in the GDP/GTP recycling rate $\kappa$. Figure 9 (left) illustrates the results of these variations. Here, we see that an increase in $\kappa$, from the base case value of 2 , corresponds to an increase in the MT polymer density $\bar{u}_{t o t}$, which is also true of the action of a MT stabilizing drug. From a biological point of view, this result makes sense. In particular, if we increase $\kappa$, we increase the rate at which free GTPtubulin is produced, which in turn promotes MT growth, similar to the action of a stabilizing MTA. Alternatively, if we decrease $\kappa$ from the base case value, we inhibit MT dynamics and MTs depolymerize at low values of $\kappa$. This result is consistent with the action of a MT destabilizing drug.

Figure 9(right) illustrates the changes in the GTP-tubulin concentration as $\kappa$ is varied. Here, it is noted that as $\kappa$ is increased, the level of free GTP-tubulin in the system does not change significantly. Thus, the GTP-tubulin is being incorporated back into the growing MTs at a faster 
beta $=10$

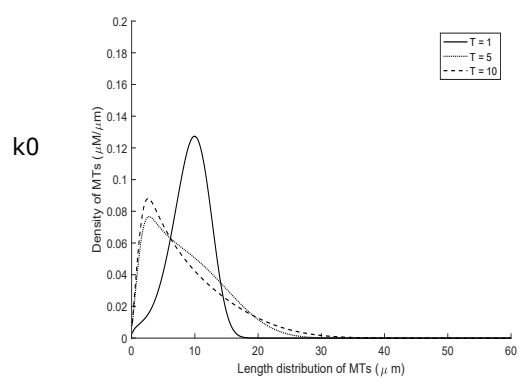

(a)

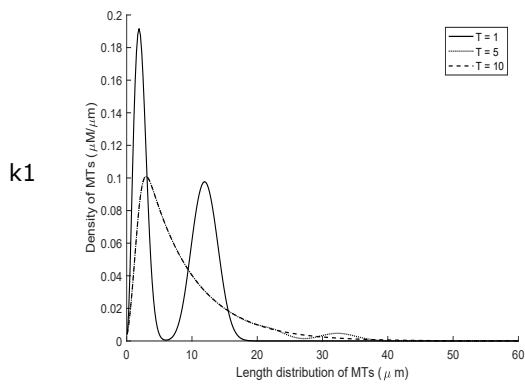

(d) beta $=30$

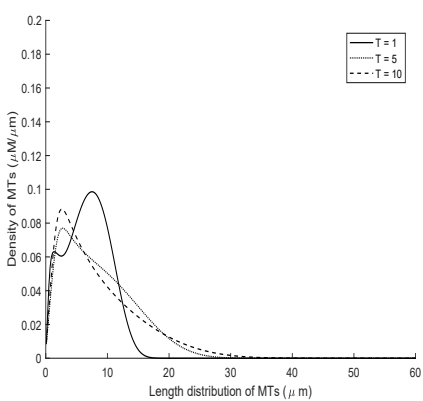

(b)

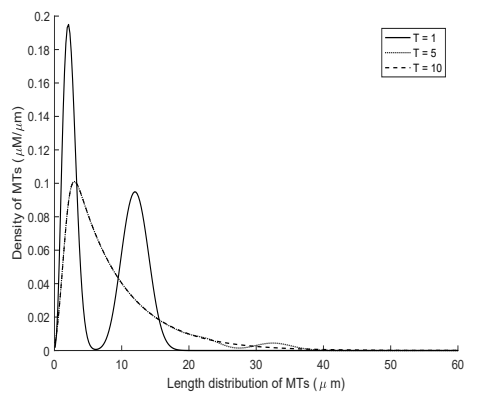

(e) superimposed long-time

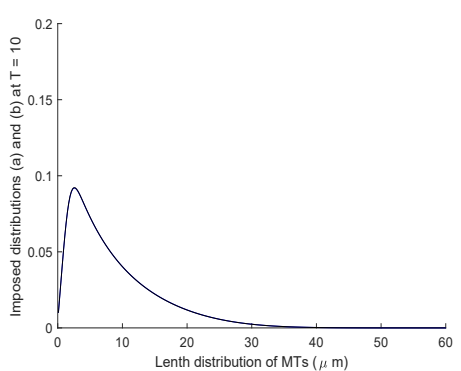

(c)

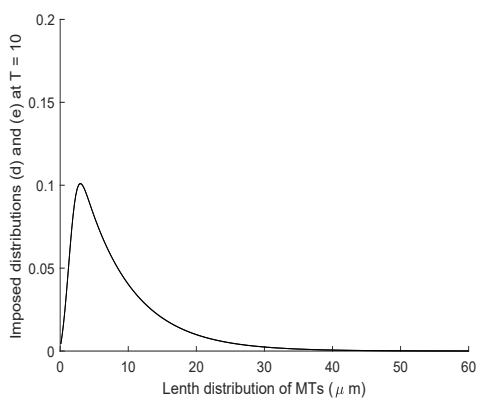

(f)

Figure 7: Examination of polymer length distribution for varying $\beta_{\infty}$ and $k_{i}$. For each $k_{i}$, the long-time length distribution is the same, regardless of the choice of $\beta_{\infty}$.

rate. Again, this promotion of polymerization is a characteristic of a MT stabilizing drug.

\subsection{Asymptotic behaviour}

In this paragraph, we consider a fragmentation kernel of type $k_{0}$ (equation $(20)$ ) with $\sigma=1$ and all the parameters given in Table 1 except for $p_{h}=6$ and the initial condition. All the plots displayed on Figure 10 are obtained for $p_{0}=11, q_{0}=0$, and four different initial size distributions IC1: $u_{0}(x)=u_{5,10}(x)$, IC2: $u_{0}(x)=u_{5,10}(x)+u_{10,10}(x)$, IC3: $u_{0}(x)=u_{10,1}(x)$, IC4: $u_{0}(x)=u_{5,1}(x)$, where

$$
u_{x_{c}, \sigma}(x)=\exp \left(-\frac{\left(x-x_{c}\right)^{2}}{\sigma}\right)
$$

and all the initial profiles being rescaled to satisfy $\int_{\mathbb{R}^{+}} x u_{0}(x) d x=1$. The numerical results suggest that the system relaxes toward an equilibrium state, i.e. that $p$ and $q$ converges toward some limiting values $p^{*}$ and $q^{*}$, and that the size distribution $u(x, t)$ converges toward an asymptotic profile $u^{*}(x)$. The limiting value for $p$ is $p^{*}=p_{h}$ and the value for $q^{*}$ (see Figure 10, upper left) and then the asymptotic profile $u^{*}$ both depend on the initial distribution $u_{0}$ (see Figure 10, upper right and Figure 11). The initial value $p_{0}$ is small enough so that there is no nucleation at time $t=0$, and the numerical simulations show that the value of $p(t)$ always stay below the threshold that triggers nucleation. As a consequence, the total number of polymers $\int_{\mathbb{R}^{+}} u(x, t) d x$ is preserved over time. The initial number of polymers is closed for IC1 and IC4 (respectively 0.197 and 0.2), and stay close for all time. Since at the beginning of the dynamic, fragmentation is turned off (i.e. $p(t) \geq p_{h}$, see Figure 10, upper left) the evolution of the system $(u, p, q)$ only depends on $p(t), q(t)$ 

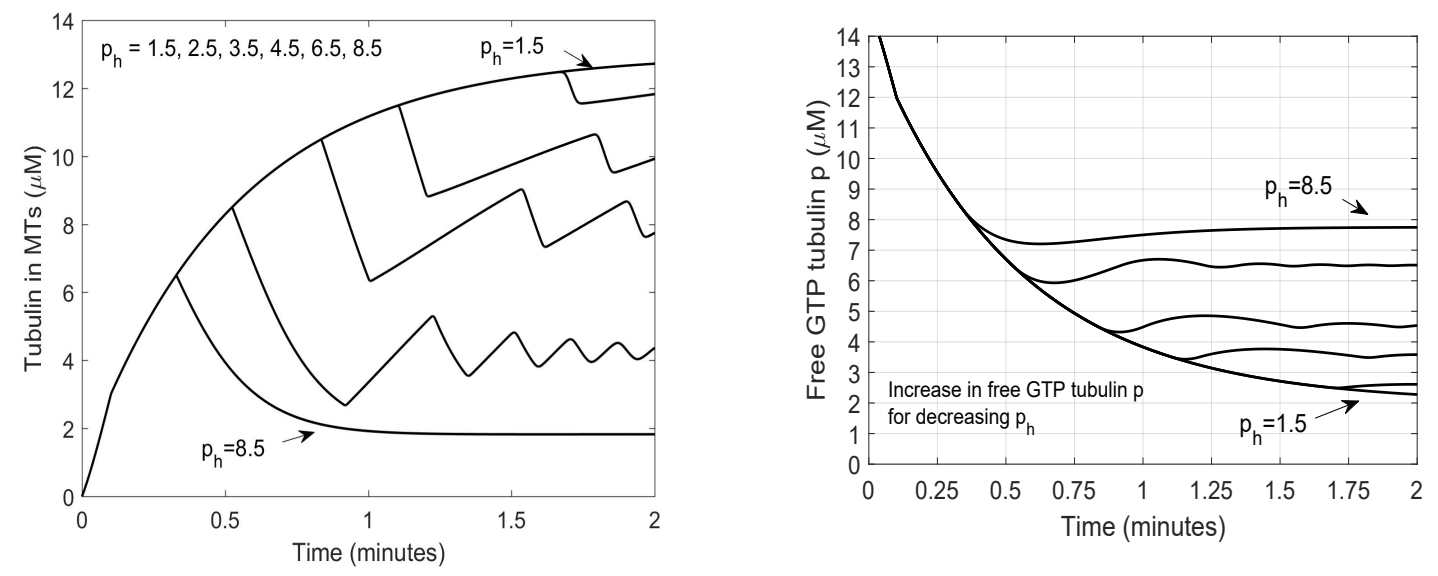

Figure 8: Left: Changes in polymerized tubulin due to variations in the hydrolysis rate. Right: Variation of the free GDP-tubulin $p(t)$ with respect to $p_{h}$.
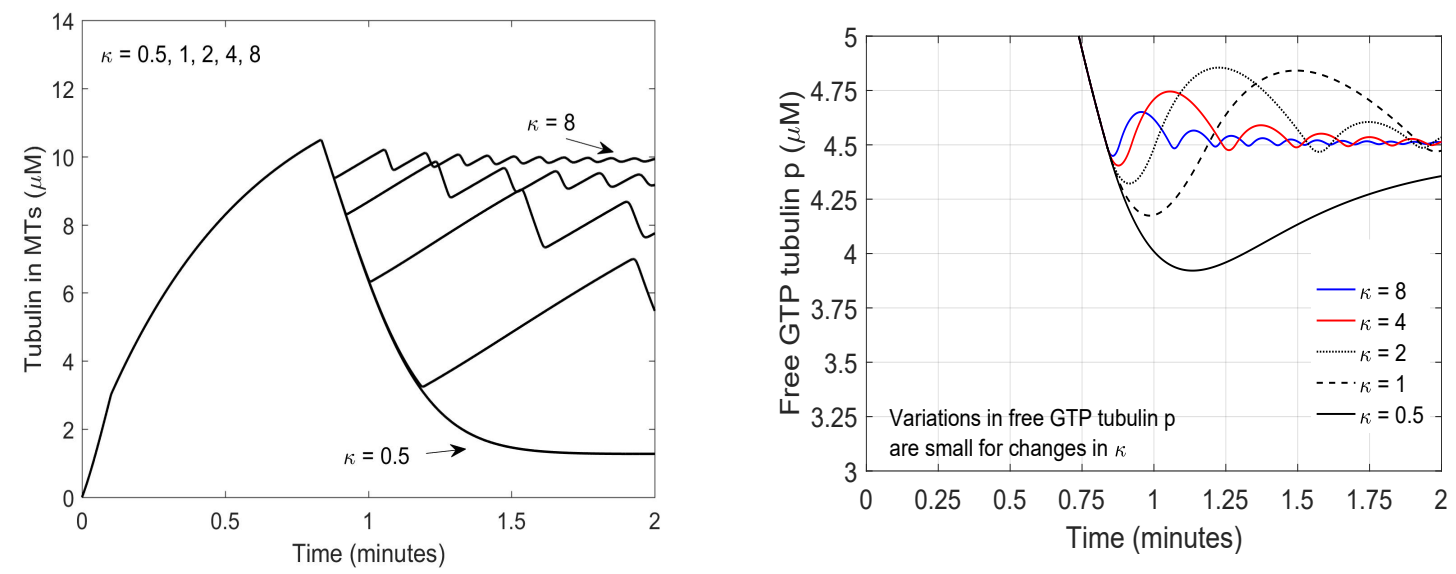

Figure 9: Left: Changes in polymerized tubulin due to variations in the the recycling rate $\kappa$. Right: Variation of the free GDP-tubulin $p(t)$ with respect to $\kappa$.

and $\int_{\mathbb{R}^{+}} u(x, t) d x$ (see system (1) (2) (3)) which explains why the time evolution of the macroscopic quantities for IC1 and IC4 are almost superimposed (Figure 10, upper left).

\section{Conclusions and perspectives}

In this paper, we have developed a novel integro-PDE modeling approach to describe MT dynamic instability. Using parameter values taken from the literature, we are able to simulate MT dynamics that are consistent with experiment. Further, through parameter variation, we are able to describe possible mechanisms for how destabilizing and stabilizing MTAs work to alter MT dynamics.

It would be interesting in future investigations to determine alternate mechanisms for how MTAs alter MT dynamics. Also, it would be interesting for biological applications to extend our model in such a way as to produce stable limit cycles (or oscillations that persist for longer periods of time). Then, because we consider a MT shortening event to occur at the time when the hydrolysis rate and the growth rate are equal, we are simplifying what is happening in real systems. In particular, in real systems MTs grow through the addition of GTP-tubulin. Once GTP-tubulin is incorporated 

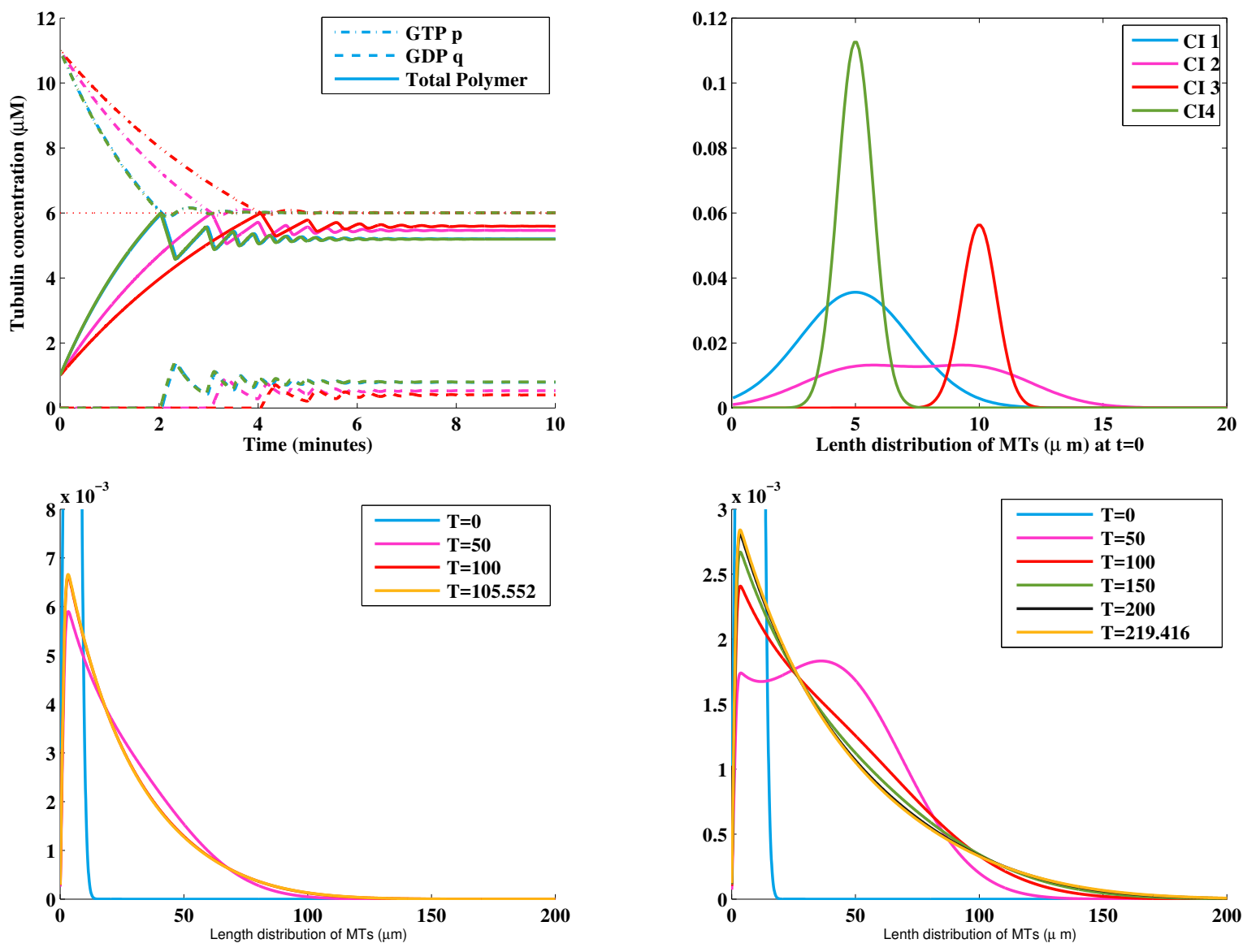

IC1

IC2

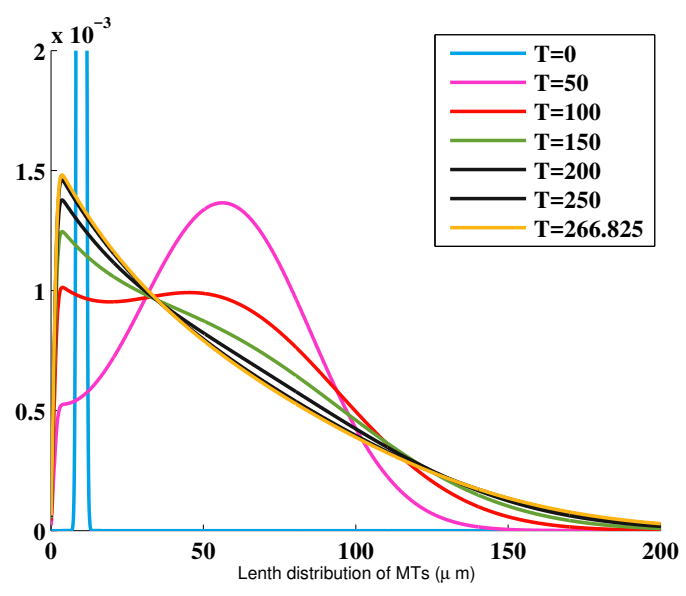

IC3

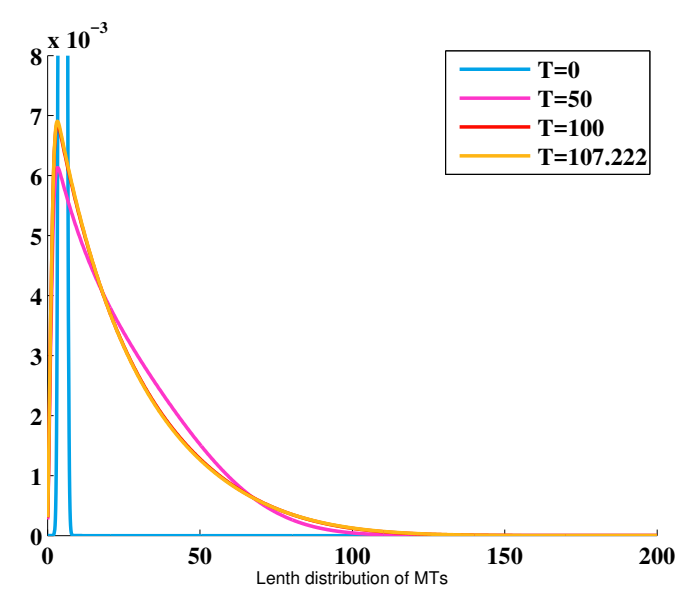

IC4

Figure 10: Upper left: Time evolution of the macroscopic quantities $p(t), q(t)$ and the mass $\int_{\mathbb{R}^{+}} x u(x, t) d x$ for different initial size distributions $u_{0}$, IC1: cyan, IC2: pink, IC3: red and IC4: green. Upper right: Four different initial size distributions $u_{0}$. On the four other plots are drawn the time evolution of the size distribution $u$ until it reaches equilibrium.

into the MT, it is hydrolyzed to lower energy GDP-tubulin, creating a distinct GTP region at the front of a MT which we call the "cap". When the hydrolysis rate is larger than the growth rate, 


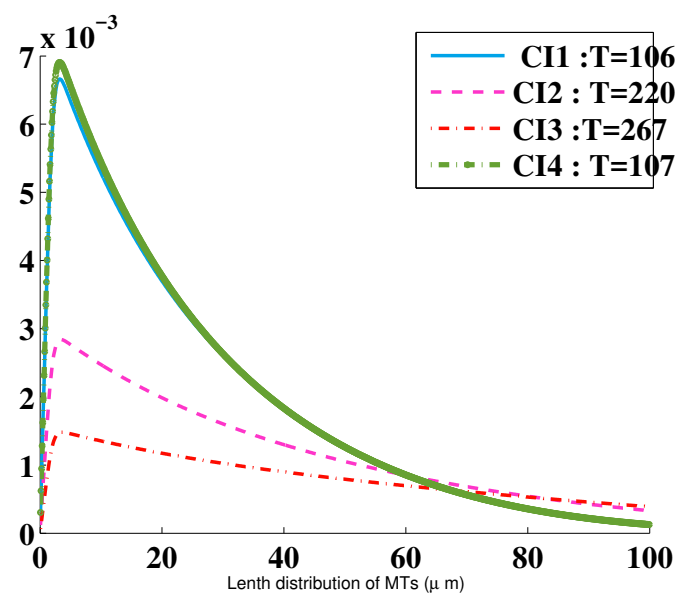

Figure 11: Asymptotic size distribution for the microtubules, for different initial size distributions $u_{0}$.

the MT cap (which we do not account for in this model) shortens. Once it disappears, then a shortening event occurs. In this modelling framework, we could account for this by incorporating a time delay at the moment in time when the hydrolysis rate becomes greater than the MT growth rate. The understanding of relaxation toward equilibrium of such systems is also a topic of interest and a future mathematical study will be carried out in that direction.

Acknowledgments. The program is funded thanks to the support of the $A^{*} M I D E X$ project $\left(n^{\circ}\right.$ ANR-11-IDEX-0001-02) funded by the "Investissements d'Avenir" French Government program, managed by the French National Research Agency (ANR), and the support INSERM Plan cancer $n^{\circ}$ PC201418. D.W. was supported by the programs cited above through a post-doc funding, F.H. and M.T. were partially supported by the programs cited above.

\section{References}

[1] P Bayley, M Schilstra, and S Martin. A simple formulation of microtubuie dynamics: quantitative implications of the dynamic instability of microtubule populations in vivo and in vitro. Journal of Cell Science, 93:241-254, 1989.

[2] A Desai and T Mitchison. Microtubule polymerization dynamics. Annual Review of Cell and Developmental Biology, 13:83-117, 1997.

[3] M Dogterom and S Leibler. Physical aspects of the growth and regulation of microtubule structures. Physical Review Letters, 70:1347-1350, 1993.

[4] H Flyvbjerg, T Holy, and S Leibler. Microtubule dynamics: caps, catastrophes, and coupled hydrolysis. Physical Review Letters E, 54:5538-5560, 1996.

[5] M Greer, L Pujo-Menjouet, and G Webb. A mathematical analysis of the dynamics of prion proliferation. J. Theoret. Biol., 242(3):598-606, 2006.

[6] T Hill and Y Chen. Phase changes at the end of a microtubule with a gtp cap. PNAS, 81(18):5772-5776, 2002. 
[7] P Hinow, V Rezania, and J Tuszynski. Continuous model for microtubule dynamics with catastrophe, rescue, and nucleation processes. Physical Review E, 80:Doi:10.1103/PhysRevE.80.031904, 2009.

[8] Stephane Honoré. Personal communication.

[9] M Kirschner and K Mitchison. Dynamic instability of microtubule growth. Nature, 312:237$242,1984$.

[10] P Laurençot and C Walker. Well-posedness for a model of prion proliferation dynamics. $J$. Evol. Equ., 7(2):241-264, 2007.

[11] H Lodish, A Berk, S Zipursky, P Matsudaira, D Baltimore, and J Darnell. Molecular Cell Biology. W. H. Freeman and Company, New York, 4 edition, 2000.

[12] I Maly. Diffusion approximation of the stochastic process of microtubule assembly. Bulletin of Mathematical Biology, 64:213-238, 2002.

[13] G Margolin, I Gregoretti, T Cickovski, C Li, W Shi, M Alber, and H Goodson. The mechanisms of a microtubule catastrophe and rescue: implications from analysis of a dimer-scale computational model. Molecular Biology of the Cell, 23, 2012.

[14] G Margolin, I Gregoretti, H Goodson, and M Alber. Analysis of a measoscopic stochastic model of microtubule dynamic instability. Physical Review E, 74, 2006.

[15] E Mukhtar, V Mustafa Adhami, and H Mukhtar. Targeting microtubules by natural agents for cancer therapy. Molecular Cancer Therapeutics, 93:275-284, 2014.

[16] A Pagano, S Honoré, R Mohan, R Berges, and A Akhmanova. Epothilone b inhibits migration of glioblastoma cells by inducing microtubule catastrophes and affecting eb1 accumulation at microtubule plus ends. Biochemical Pharmacology, 84:432-443, 2012.

[17] B Perthame. Transport equations in biology. Frontiers in Mathematics. Birkhäuser Verlag, Basel, 2007.

[18] B Perthame. Parabolic equations in biology. Lecture Notes on Mathematical Modelling in the Life Sciences. Springer, Cham, 2015. Growth, reaction, movement and diffusion.

[19] D Sept, H Limbach, H Bolterauer, and J Tuszynski. A chemical kinetics model for microtubule oscillations. Journal of Theoretical Biology, 197:77-88, 1999.

[20] G Simonett and C Walker. On the solvability of a mathematical model for prion proliferation. J. Math. Anal. Appl., 324(1):580-603, 2006.

[21] R Wade. On and around microtubules: An overview. Molecular Biotechnology, 43:177-191, 2009.

[22] C Walker. Prion proliferation with unbounded polymerization rates. In Proceedings of the Sixth Mississippi State-UBA Conference on Differential Equations and Computational Simulations, volume 15 of Electron. J. Differ. Equ. Conf., pages 387-397. Southwest Texas State Univ., San Marcos, TX, 2007. 
[23] R Walker, E O'Brien, N Pryer, M Soboeiro, W Voter, H Erickson, and E Salmon. Dynamic instability of individual microtubules analyzed by video light microscopy: Rate constants and transition frequencies. Journal of Cell Biology, 107:1437-1448, 1988.

[24] D White, F Hubert, and S Honoré. Exploring the effect of end-binding proteins and microtubule targeting chemotherapy drugs on microtubule dynamic instability. Journal of Theoretical Biology, 429:18-34, 2017.

[25] E Zauderer. Partial Differential Equations of Applied Mathematics. John Wiley and Sons, Inc. New Jersey, 3 edition, 2006.

[26] J Zhou and P Giannakakou. Targeting microtubules for cancer chemotherapy. Current Medical Chemistry, 5:65-71, 2005. 